\title{
IPAC2010 Accelerator Prize article: Particle accelerators and colliders
}

\author{
Stephen Myers \\ CERN, CH-1211 Geneva 23, Switzerland \\ ADAM SA Rue de Veyrot, CH-1217 Meyrin, Geneva, Switzerland
}

(Received 10 September 2020; accepted 10 November 2020; published 11 December 2020)

\begin{abstract}
In this document, I recall my own personal memories of the conception, design, construction and operation of particle accelerators and particle colliders over the past 50 years. This is not meant to be a technical report, and this is evidenced by the style of writing (commonly used first person singular), but more to give an insight to the historical beginnings and endings of some of the world's most technically complex and expensive scientific instruments.
\end{abstract}

DOI: 10.1103/PhysRevAccelBeams.23.124802

\section{PARTICLE PHYSICS AND ACCELERATORS}

High energy particle accelerators are fundamental tools for investigating the origins of the Universe. The most important parameter in the design of any type of particle accelerator is the beam energy available for the production of new particles. Analogically we can consider particle accelerators as large "microscopes," the spatial resolution of which increases with beam energy. The "history of the Universe" is depicted in the diagram in Fig. 1 where the evolution of the Universe is shown as a function of three parameters: (i) " $\mathrm{t}$ ", time starting with the "big bang" around 13.8 billion years ago, (ii) " $\mathrm{T}$ " absolute temperature with unimaginable initial temperatures $\left(10^{32}\right.$ Kelvin $)$ which gradually cooled down to $2.7 \mathrm{~K}$ (around -270 Celsius) over 13.8 billion years, and (iii) "E" energy in the system shown in $\mathrm{GeV}\left(10^{9} \mathrm{eV}\right)$, the unit of energy which is quoted for most high energy accelerators $(1 \mathrm{TeV}=$ $1000 \mathrm{GeV}=10^{12} \mathrm{eV}$ ).

The diagram shows an artist's view of the existence of various types of particles (electrons, quarks, bosons, etc.) as a function of the three parameters, $t, T$, and $E$.

If we now think of particle accelerators with increasing energy available for the production of new particles, this is depicted by the yellow arrow.

By accelerating to high energies (along the yellow line) we are recreating in a small controlled way the conditions which existed at this point in time. The higher the available energy, the closer we get to creating some of the phenomena which existed at that particular instant after the big bang.

Published by the American Physical Society under the terms of the Creative Commons Attribution 4.0 International license. Further distribution of this work must maintain attribution to the author(s) and the published article's title, journal citation, and DOI.
Also shown in the diagram (upper left) is superimposed the available energy of recent accelerators and colliders.

\section{A. General characteristics of particles to be accelerated}

It should now be clear that, in order to be able to probe to conditions which existed just after the creation of the Universe, we need to generate higher and higher energies available for the production of new phenomena and particles. Since acceleration of particles needs a certain amount of time, it is also clear that we should accelerate particles (or antiparticles) which are stable or have a long half-life. In addition, it is also obvious that the particles must be charged to allow acceleration, bending and focusing by electromagnetic fields. The most common particles used in accelerators are electrons, positrons (antielectrons), protons, antiprotons and some charged heavy ions like lead, gold, copper etc.

\section{HIGH ENERGY ACCELERATORS}

Lord Rutherford was the "god-father" of accelerators. In his inaugural presidential address to the Royal Society in London in 1928, he said [1] "I have long hoped for a source of positive particles more energetic than those emitted from natural radioactive substances." This was the start of a long quest for the production of high energy beams of particles in a very controlled way. Just three years later, the proof of concept of the cyclotron, invented and patented by Ernest Lawrence [2], was demonstrated by the "9-inch cyclotron" at the University of California, Berkeley. This was the birth of big science and cyclotrons of larger size and higher energy quickly followed. During the ensuing eleven years a further six cyclotrons were built at Berkeley, the largest being the 184-inch cyclotron which began operation in 1942 and was operated for more than 50 years [3]. The 37-inch and later the 184-inch cyclotron magnet were transformed into mass spectrographs for separating uranium-235 from -238. In 1942 Lawrence and his team 


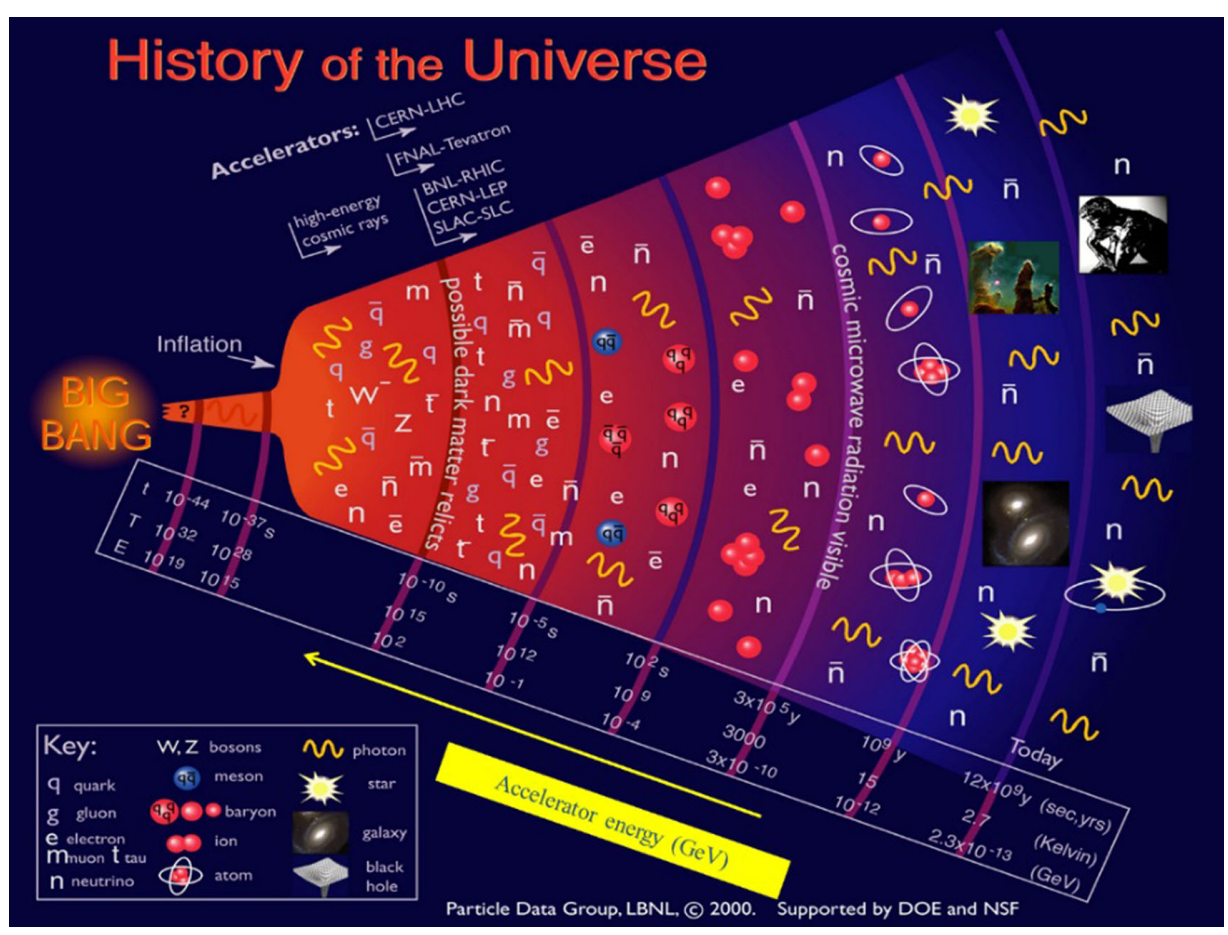

FIG. 1. History of the Universe.

had developed a successful prototype mass spectrograph which was called the "calutron" named after the University of California. Nearly all of the uranium-235 in the atomic bomb that destroyed Hiroshima was generated using Lawrence's calutrons.

From 1949 until 1952, the elements 97 (berkelium) to 100 (fermium) were discovered at the University of California, Berkeley, using Lawrence cyclotrons.

Following the end of World War II "nuclear" physicists were held in very high esteem since they had developed a nuclear bomb which brought an early end to the War. The political admiration for nuclear was used in the creation of CERN in 1954 by naming the new organization the European Council for Nuclear research (in French; Conseil Européen pour la Recherche Nucléaire).

Following the War, the 88- and the 184-inch cyclotrons continued to be used for the production and study of many radioisotopes. These devices accelerated protons, deuterons and ions from hydrogen to uranium. and were even used for performing the first "single event upsets" electronics radiation experiments as well as testing U.S. spacecraft materials. As the beam energy demands from high energy physics experiments increased, the cyclotron concept became much too large and costly and for these higher energies, a new type of high energy accelerator was invented called the synchrotron.

\section{A. The synchrotron}

The principle of the synchrotron was invented in 1944 by Vladimir Veksler. Ed McMillan arrived at the same idea independently in 1945 having missed the Veksler publication which although published in English was only available in the Soviet Union. However, one year earlier in 1943, Australian scientist, Marcus Oliphant (who was at Oakridge, USA at the time) proposed the idea of a pulsed magnet ring, which is fundamental to the principle of the synchrotron, see for example [4].

The paper by McMillan to the editor of Physics Review used the terms "phase stability" and "synchrotron." Phase stability is the essential ingredient in particles being accelerated in a synchrotron. When the conditions are correctly set up, the accelerating fields produce "potential wells" (also called accelerating "buckets") inside which the charged particles oscillate stably in energy and phase with respect to the synchronous particle. Effectively the potential well creates "bunches" of the charged particles and allows them to be accelerated. The motion of a single particle inside the accelerating potential well is analogous to a child on a garden swing. In the absence of friction, the amplitude of the swing oscillations would remain constant as the child swings to and fro. In the case of the swing the potential well allows oscillations only as high as the rope support of the swing. Motion amplitudes greater that the support would cause the child to be outside the potential well and fall down. Likewise, particles outside the rf bucket will not be accelerated.

McMillan led the construction of the first electron synchrotron in $1945(300 \mathrm{MeV})$ and the first proton synchrotron was designed and built by Marcus Oliphant in 1953 (University of Birmingham). 
The synchrotron has the advantage over the cyclotron that the magnetic fields need only be present over the actual region of the particle orbits, thereby reducing hugely the magnet costs. In synchrotrons the particles are accelerated on every revolution by pulsed electromagnetic fields, and the increase in energy is matched by increases in the magnetic fields in synchronism with the changes in revolution time.

The next major invention was the discovery of strong (or alternating gradient) focusing. Courant, Livingstone, and Snyder published the paper on strong focusing near the end of 1952 [5] only to find that the idea had already been conceived in 1949 and patented by Christofilos [6]. The magnetic strong focusing is the optical analogy of optical focusing with equal concave and convex lenses. The magnetic strong focusing produces beams of much smaller cross sections and hence smaller magnet apertures and smaller vacuum chambers. In CERN, this recent development initiated a change in the design of the $10 \mathrm{GeV}$ weak focusing machine for a $26 \mathrm{GeV}$ strong focusing synchrotron called the CERN Proton Synchrotron (PS). At the same time Brookhaven has also planned their next machine, the $33 \mathrm{GeV}$ AGS.

By 1959 CERN's Proton Synchrotron (CPS) was ready for commissioning, one year ahead of Brookhaven's
Alternating Gradient Synchrotron (AGS) and had, as feared, difficulties crossing "transition energy" with the new alternating gradient optics. Then Wolfgang Schnell produced a circuit which he had built in a Nescafe tin, to jump the rf phase at the moment of transition. Schnell, his box and a few connectors brought the beam through transition to full energy.

As of 2020, the CPS and the AGS have been operating successfully and reliably for the past 60 years. The PS has been the injector for the CERN Intersecting Storage Rings (ISR), the Super Proton Synchrotron (SPS), the super proton-antiproton collider (SPPSS), the large electron-positron collider (LEP), and the Large Hadron Collider (LHC) as well as providing beams for its own experimental areas such as neutron time of flight (n-TOF), fixed target experiments in the east area, and the antiproton facilities (see Fig. 2). The AGS has been the injector of heavy ions and polarized protons for RHIC.

\section{B. Beam energy}

Over the past century the quest for higher and higher beam energies has been achieved by technical breakthroughs, and invention of different types of accelerators.

\section{The CERN accelerator complex Complexe des accélérateurs du CERN}

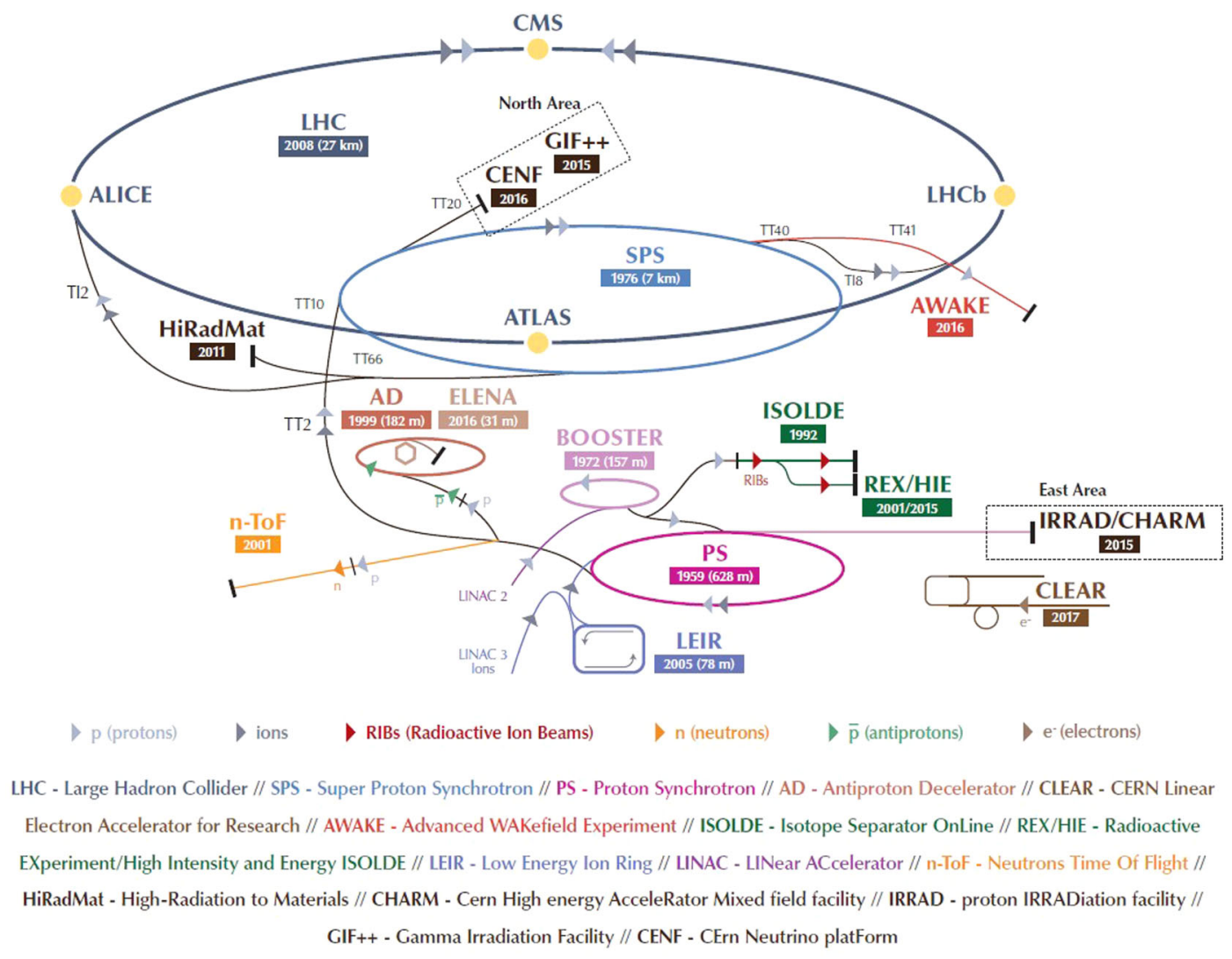

FIG. 2. Schematic of CERN accelerator complex. 
In many cases the accelerator requirements have been the driving force behind some of the new technologies, as well as producing spin-off into other fields of research. This sustained increase was also due to particle "colliders" where one beam is brought into collision with another beam coming in the opposite direction.

For a fixed target experiment, and neglecting the mass of the target, the energy available for physics production increases with the square root of the incident energy, whereas for colliders the "center of mass" energy is equal to the sum of the energies of the two beams. As an example, the $6.5 \mathrm{TeV}$ beam of the LHC impinging on a stationary target produces "only" a physics production energy of $110 \mathrm{GeV}$, a factor of nearly 120 times lower that the $13000 \mathrm{GeV}$ center of mass collision energy of the LHC.

In the quest for new discoveries, finding ways to increase the production energy became of paramount importance and attention was firmly focused on colliders.

\section{Colliders}

It was realized that although colliders had the potential for very high production energies, the technology would be much more difficult. Firstly, there were concerns about the production rate due to the fact that the density of the

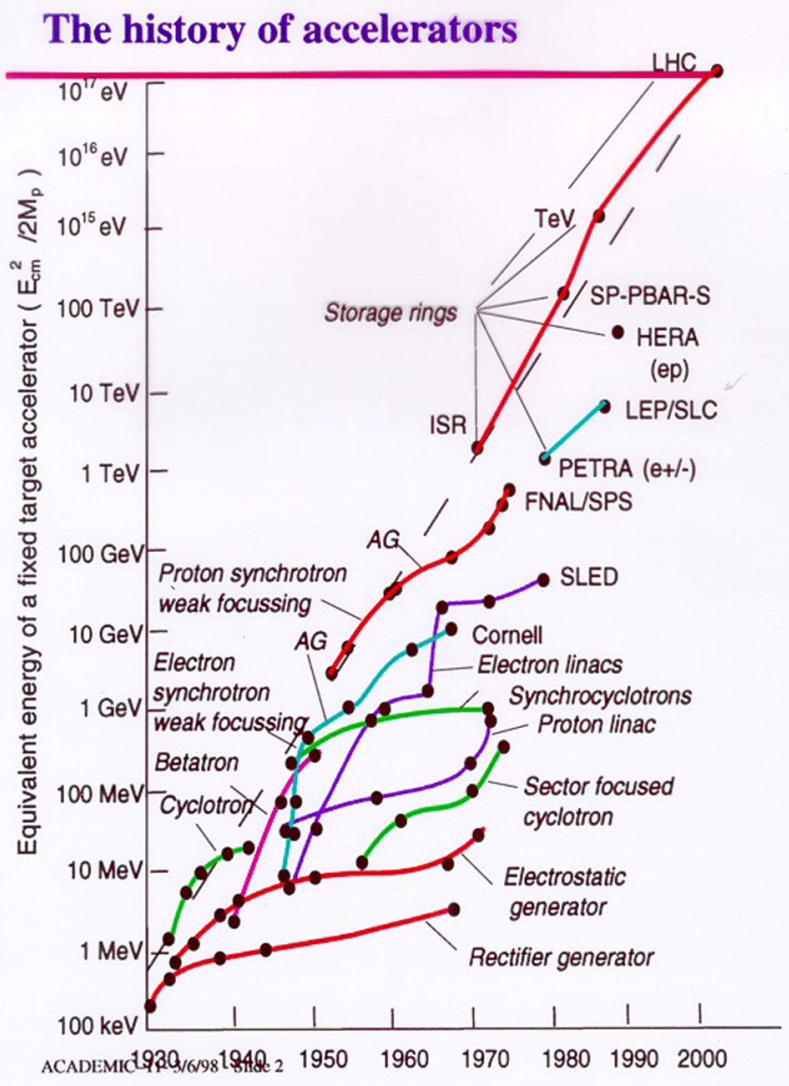

FIG. 3. Energy evolution of particle accelerators: Livingston chart, first published by Livingston [7]. oncoming beam was many orders of magnitude less than that of a solid target. Therefore, the bunches had to interact many times per second (circular collider), and the density at the collision points had to be maximized (high beam intensities and tiny beam cross-section areas). As mentioned before, this approach only works with stable particles.

Furthermore, for a beam to survive circulating for a long time, it is necessary to reduce beam-gas interactions, which requires an ultrahigh vacuum system for the beam chamber (typically lower than $10^{-9} \mathrm{~Pa}$ ).

Another feared disadvantage with the move to colliders was that fixed target installations were very flexible and allowed multiple different experiments by sharing the beams in separate beam lines at various energies and even with different types of particles. The schema of the CERN accelerator complex demonstrates this, where the PS as an example, feeds many different experiments on a "pulse-topulse" basis, as well as being one of the LHC injectors. In contrast, colliders had a limited number of interaction points.

\section{Livingstone chart}

The "Livingston" [7] chart in Fig. 3 shows the exponential increase in the available energy for collisions as a function of time.

\section{WHICH PARTICLES TO COLLIDE?}

\section{A. Single ring colliders}

The next point of debate for colliders was the choice of the types of particles to collide. The advantage of colliding particles with their antiparticle (e.g. electrons against positrons) was that only a single ring was needed since for example, electrons traveling clockwise in a magnetic field are bent and focused in the same way as positrons traveling anticlockwise. This simple fact meant that e-p colliders only needed a single set of magnets and a single vacuum chamber. This is an enormous economic and technical advantage. The huge disadvantage is the number of bunches which can be circulated in each beam. It is clear that with a single bunch circulating in each direction they will collide at two equidistant points around the circumference. So, with $\mathrm{n}$ bunches of particles per beam there are $2 \mathrm{n}$ collision points. Colliding at many points has a very detrimental effect on the lifetime of the beams due to the nonlinear "beam-beam" effect and limiting the number of bunches limits the total number of interactions per second.

\section{B. Two ring colliders}

If the collider has two separate rings, then the beams can be electromagnetically steered into collisions at a predefined number of locations thereby avoiding the problem with beam lifetime with a large number of bunches. The disadvantage here is cost and technical complexity 
increased by a straight factor of up to 2 . However, with two separate rings the collision rate can be significantly increased.

\section{Synchrotron radiation}

It therefore became fairly clear that colliders could use leptons (electrons and positrons) and hadrons (protons, antiprotons, and heavier ions). The effect of "synchrotron radiation" plays an important role in all lepton colliders. When an energetic charged particle is subjected to a magnetic field which is perpendicular to its direction of motion, then due to the transverse deceleration, quantum photons are emitted which reduce the energy of the particle. This is similar to the way $\mathrm{x}$ rays are produced in an $\mathrm{x}$-ray tube where electrons are quickly decelerated by bombarding the tungsten anode. In a circular accelerator the average energy loss per turn of the primary particle is proportional to the fourth power of the relative energy of the particle and inversely proportional to the magnet bending radius.

The rest mass $\left(\mathrm{E}_{0}\right)$ of an electron is $0.511 \mathrm{MeV}$ and the rest mass of a proton is $938.272 \mathrm{MeV}$ i.e. a factor of 1836 greater. Consequently, electrons at the same absolute energy as protons $(E)$ have a relative energy $\left(E / E_{0}\right)$ a factor of 1836 times higher. This means that the average energy loss due to synchrotron radiation emission by electrons (at the same energy as protons) is $1.1 \times 10^{13}$ times more than that of the proton!!

This energy loss is replenished by the rf acceleration system which is synchronized to the circulating beam to give electromagnetic accelerations ("kicks"), always in the correct direction on each revolution of the particles. Clearly for very high electron/positron energies, the synchrotron radiation energy losses become enormous which in turn requires very high voltage acceleration systems. As an example, the energy loss per turn in the energy upgraded LEP collider (LEP2) at $104 \mathrm{GeV}$ was $\sim 3 \mathrm{GV}$, which required that the rf system operated at $3.4 \mathrm{GV}$ per turn.

The amount of beam power lost (MW) is proportional to the product of the energy loss per turn $(\mathrm{eV})$ and the average beam current. In LEP2, the radiated synchrotron power at $104 \mathrm{GeV}$ was close to $20 \mathrm{MW}$. The synchrotron radiation power emitted can damage material around the ring and must be fed back to the beam via the rf system.

These huge numbers indicate that synchrotron radiation imposes a severe limitation on the maximum energy of electron-positron colliders. With the closure of LEP2 in 2000, it was generally accepted that LEP2 would be the highest energy electron-positron collider ever to be built. This is no longer true with the proposal for TLEP (more recently renamed FCC electron-positron option) as part of the Future CERN Collider (FCC) project.

The combination of the quantum radiation losses and the rf system energy replenishment produces an effect called synchrotron damping. The combination of synchrotron damping and quantum "heating" results in a statistical equilibrium for the beam dimensions. If the beam is subjected to small perturbations which change its dimensions, it will return to its equilibrium size in a time given by the damping time. The net result of this is that lepton stored beams are almost impervious to external perturbations.

This is a remarkable feature for lepton storage rings even at relatively low energy. However, it is also clear that due to the strong dependence of synchrotron radiation on beam energy there is a practical and economic limitation for electron storage rings at very high energies due to the required installed rf. This limitation (almost) does not exist for hadron storage rings. However, due to the vanishing amounts of synchrotron damping with hadron storage rings, all perturbations to a proton beam will be "remembered" for the lifetime of the beam. This fact led to the fear that hadron storage rings would never be able to operate for long periods of time. This fear was proven to be unjustified by the operation of the CERN Intersecting Storage rings.

\section{The beam-beam effect}

By definition, in storage ring colliders, the beams are brought into collision in order to produce the high energy interactions. Since the particles are charged, there is an electromagnetic $(\mathrm{e}-\mathrm{m})$ field associated with each beam which acts on the counterrotating beam. Consequently, each particle in one of the beams experiences the e-m field of the other beam. The strength of this e-m field is dependent on the distance between the affected particle and the center of the opposing beam. For small displacements from the center, the dependence on distance is linear whereas as the distance becomes greater the field is highly nonlinear. The slope of the linear dependence is identical to a focusing (or defocusing) magnet and is called the beambeam tune shift. Early experience revealed that the performance of colliders was severely degraded by the nonlinear beam-beam effect. For all colliders there is an upper limit to the value of the beam-beam tune shift which could be reached before degradation of performance. The beambeam effect is one of the most fundamental limitations to the production performance of all colliders.

\section{E. Risks related to very high energy superconducting hadron colliders}

For the reasons previously pointed out, present highest energy hadron colliders are not strongly affected by synchrotron radiation. The maximum energy of a hadron collider is directly proportional to the maximum strength of the magnetic bending fields and the circumference of the ring (bending radius). The size of the rings imposes a financial, and geological limit whereas the maximum magnetic field imposes a severe technical limit. For all recent hadron colliders, superconducting magnets have been used to maximize the beam energy. The development of superconducting magnets has been a major contributor 
to the advances made in increasing the beam energy for hadron colliders. The advantages of superconductivity are the much higher magnetic fields which can be achieved, and the low amount of electrical power consumption needed. These advantages do not come free of charge.

\section{Stored energy, quench and magnet protection}

In a superconducting (sc) magnet, the high magnetic fields are produced by the large currents flowing through the superconducting coils. These sc magnet coils also present a large electrical inductance $(L)$. The stored energy

\begin{tabular}{|c|c|c|c|c|c|c|}
\hline & Species & $E_{b}, \mathrm{GeV}$ & $C, \mathrm{~m}$ & Lmax peak & Years & $\begin{array}{c}\text { SM } \\
\text { Participation }\end{array}$ \\
\hline $\mathrm{AdA}$ & $e+e-$ & 0.25 & 4.1 & 1025 & 1964 & $\mathrm{~N}$ \\
\hline VEP-1 & $e-e-$ & 0.16 & 2.7 & $5 \times 10^{27}$ & 1964-68 & $\mathrm{N}$ \\
\hline CBX & $e-e-$ & 0.5 & 11.8 & $2 \times 10^{28}$ & 1965-68 & $\mathrm{N}$ \\
\hline VEPP-2 & $e+e-$ & 0.67 & 11.5 & $4 \times 10^{28}$ & 1966-70 & $\mathrm{N}$ \\
\hline ACO & $e+e-$ & 0.54 & 22 & 1029 & $1967-72$ & $\mathrm{~N}$ \\
\hline ADONE & $e+e-$ & 1.5 & 105 & $6 \times 10^{29}$ & $1969-93$ & $\mathrm{~N}$ \\
\hline CEA & $e+e-$ & 3 & 226 & $0.8 \times 10^{28}$ & 1971-73 & $\mathrm{N}$ \\
\hline ISR & $p p$ & 31.4 & 942 & $1.4 \times 10^{32}$ & 1971-80 & YYY \\
\hline SPEAR & $e+e-$ & 4.2 & 234 & $1.2 \times 10^{31}$ & $1972-90$ & $Y$ \\
\hline DORIS & $e+e-$ & 5.6 & 289 & $3.3 \times 10^{31}$ & 1973-93 & $Y$ \\
\hline VEPP-2M & $e+e-$ & 0.7 & 18 & $5 \times 10^{30}$ & $\begin{array}{l}1974- \\
2000 \\
\end{array}$ & $\mathrm{~N}$ \\
\hline VEPP-3 & $e+e-$ & 1.55 & 74 & $2 \times 10^{27}$ & 1974-75 & $\mathrm{N}$ \\
\hline DCI & $e+e-$ & 1.8 & 94.6 & $2 \times 10^{30}$ & 1977-84 & $\mathrm{N}$ \\
\hline PETRA & $e+e-$ & 23.4 & 2304 & $2.4 \times 10^{31}$ & 1978-86 & $Y Y$ \\
\hline CESR & $e+e-$ & 6 & 768 & $1.3 \times 10^{33}$ & $\begin{array}{l}1979- \\
2008\end{array}$ & $Y$ \\
\hline PEP & $e+e-$ & 15 & 2200 & $6 \times 10^{31}$ & $1980-90$ & $Y Y$ \\
\hline $\mathrm{Spp}^{-\mathrm{S}}$ & $p p^{-}$ & 455 & 6911 & $6 \times 10^{30}$ & 1981-90 & $Y Y$ \\
\hline TRISTAN & $e+e-$ & 32 & 3018 & $4 \times 10^{31}$ & 1987-95 & $Y$ \\
\hline Tevatron & $p p^{-}$ & 980 & 6283 & $4.3 \times 10^{32}$ & $\begin{array}{c}1987- \\
2011 \\
\end{array}$ & $Y$ \\
\hline SLC & $e+e-$ & 50 & 2920 & $2.5 \times 10^{30}$ & 1989-98 & $Y$ \\
\hline LEP & $e+e-$ & 104.6 & 26567 & 1032 & $\begin{array}{l}1989- \\
2000\end{array}$ & YYY \\
\hline HERA & $e p$ & $30+920$ & 6336 & $7.5 \times 10^{31}$ & $\begin{array}{l}1992- \\
2007 \\
\end{array}$ & YY \\
\hline PEP-II & $e+e-$ & $3.1+9$ & 2200 & $1.2 \times 10^{34}$ & $\begin{array}{l}1999- \\
2008 \\
\end{array}$ & $N$ \\
\hline KEKB & $e+e-$ & $3.5+8.0$ & 3016 & $2.1 \times 10^{34}$ & $\begin{array}{l}1999- \\
2010 \\
\end{array}$ & $\mathrm{~N}$ \\
\hline VEPP-4M & $e+e-$ & 6 & 366 & $2 \times 10^{31}$ & 1979- & $\mathrm{N}$ \\
\hline BEPC-I/II & $e+e-$ & 2.3 & 238 & 1033 & 1989- & $Y$ \\
\hline DAФNE & $e+e-$ & 0.51 & 98 & $4.5 \times 10^{32}$ & 1997- & $Y Y$ \\
\hline RHIC & $p, i$ & 255 & 3834 & $2.5 \times 10^{32}$ & 2000- & $Y$ \\
\hline LHC & $p, i$ & 6500 & 2669 & $2.1 \times 10^{34}$ & $2009-$ & YYY \\
\hline VEPP2000 & $e+e-$ & 1 & 24 & $4 \times 10^{31}$ & 2010- & $\mathrm{N}$ \\
\hline S-KEKB & $e+e-$ & $7+4$ & 3016 & $8 \times 1035 *$ & 2018- & $Y$ \\
\hline
\end{tabular}

FIG. 4. Particle colliders from Shiltsev and Zimmermann: arxiv:2003.09084. 
in an inductor is $0.5 I^{2} L$, where $I$ is the current (A) and $L$ the inductance $(\mathrm{H})$. Consequently, in sc magnets there is an inherent amount of stored electrical energy.

The large currents can flow without resistance due to the phenomenon of superconductivity: i.e. in sc coils the resistance approaches zero when the coil is sufficiently cold, typically below around 4 kelvin $(-269 \mathrm{C})$, meaning no resistive losses. If the coil temperature increases above its critical temperature, the coil becomes resistive, and initiates an avalanche process called a "quench." In a quench, the coil begins to be heated by some effect (frictional movements, proton losses, etc.) and this extraneous heating increases the resistance of the coil, consequently additional heating is produced due to the current flowing in a now resistive coil.

Superconducting magnets are protected against damage resulting from quenches by a magnet protection system. The magnet protection system measures the resistance of the coils and if the resistance increases, the stored energy in the magnets is transferred to an energy "dump" thereby avoiding damage to the magnet.

In the LHC, at maximum field, the current flowing in the coils $(I)$ of the superconducting magnets is around $12 \mathrm{kA}$ and the total inductance is more than $120 \mathrm{H}$. This produces a huge amount of stored energy: 11 gigajoules, about the same as a Nimitz-class aircraft carrier going at full battle speed, or the energy needed to heat up and melt more than 16 tons of copper. The electrical stored energy in superconducting magnets introduces an increased risk factor for the operation of such systems. This high risk was demonstrated in September 2008 with the accident in the LHC (see later).

\section{Stored energy in the beams}

The definition of energy in Joules: 1 Joule is the energy required to raise 1 Coulomb by 1 Volt:

$$
E=Q V \text {. }
$$

For the design energy of the LHC $(7 \mathrm{TeV})$ proton charge $=1.6 \times 10^{-19} \mathrm{C}, 1.15 \times 10^{11}$ protons per bunch and 2808 bunches, the total energy stored in the beam is
362 MJ. Knowing that the energy needed to heat up and melt $1 \mathrm{~kg}$ of copper is $0.73 \mathrm{MJ}$ leads to the fact that the beam in the LHC has enough energy to melt nearly $500 \mathrm{~kg}$ of copper. The beam in the LHC is steered and focused by the control of many thousands of components. If any one of these components fails, then the beam will be "out of control." For this reason, the equipment surrounding the LHC beam (as it circulates for many hours) must be protected against the destructive energy of the beam. This "machine protection" system must detect any failure and abort the beam before it can destroy the surrounding components. The beam is aborted into a tunnel tangential to the beam tunnel and steered onto a beam dump. The beam protection system must never fail otherwise many components will be destroyed.

This is the second major risk associated with very high energy hadron colliders.

\section{HISTORY OF ACCELERATORS}

In 1961, the first collider built (the 1.3-meter diameter electron-positron AdA) was operated with a low intensity beam of electrons at $250 \mathrm{MeV}$ in Frascati, Italy. See for example [8]. It was a further ten years before the ISR was operated in 1971.

Figure 4 summarizes the details of all colliders ever operated with beam. On the further most right-hand column is indicated the colliders in which I personally participated either (on the CERN colliders) where I had direct responsibilities, or as external advisor.

\section{50 YEARS OF COLLIDERS: A PERSONAL PERSPECTIVE}

\section{A. ISR first $\mathbf{p p}$ and $\mathbf{p} \overline{\mathbf{p}}$ collider}

\section{Introduction}

In 1956, during the construction of the CERN synchrocyclotron and the PS, studies for the second generation of CERN accelerators began and gradually converged towards a proton-proton collider. In addition, from 1961 onwards, a study of a $300 \mathrm{GeV}$ proton synchrotron was carried out. It was decided to construct the ISR first, even though a number of physicists were not in favor as the ISR would not

TABLE I. Basic parameters of the ISR.

\begin{tabular}{lc}
\hline \hline Colliding particles & pp, dd, pd, $\alpha \alpha, \alpha \mathrm{p}, \mathrm{ppbar}$ \\
Particle momentum & 3.5 to $31.4 \mathrm{GeV} / c$ \\
Circumference $(\mathrm{m})$ & $942.5 \mathrm{~m}(300 \pi)$ \\
Number of main magnets & $132 / \mathrm{ring}$ \\
Magnetic dipole field & $1.33 \mathrm{~T}(\mathrm{max})$ \\
Length of main magnets & $4.88 / 2.44 \mathrm{~m}$ \\
Betatron oscillations/turn & $8.9(\mathrm{~h}), 8.88(\mathrm{v})$ \\
$\beta *(\mathrm{~h} / \mathrm{v})$ & $21 \mathrm{~m} / 12 \mathrm{~m}$ \\
$\beta *(\mathrm{~h} / \mathrm{v})$ & $2.5 \mathrm{~m} / 0.28 \mathrm{~m} \mathrm{in} \mathrm{sc} \mathrm{low-beta} \mathrm{section}$ \\
rf system per ring & 7 cavities, $9.5 \mathrm{MHz}, 16 \mathrm{kV}$ rf peak voltage \\
\hline \hline
\end{tabular}


provide secondary beams and a collider project was considered to be very risky.

In June 1965, the CERN Council approved the construction of the ISR facility, and, at its meeting in December of the same year, accepted the financial plan of the project and to commence funding in January 1966. The construction budget was 332 million CHF (1965 value) and first operation was foreseen for mid-1971.

The CERN Intersecting Storage Rings (ISR) was the first proton-proton collider ever constructed and operated. The ISR proceeded as planned with first beams in 1971 and operation for physics from 1971 to 1983.

Table I gives the basic parameters of the storage rings.

The ISR consisted of two independent storage rings intersecting at eight points with a crossing angle of 14.8 degrees (see Figs. 5 and 6). The circumference of the rings was 943 meters ( 1.5 times that of the CERN Proton Synchrotron which supplied particles to the ISR). The larger circumference was needed to allow space for the

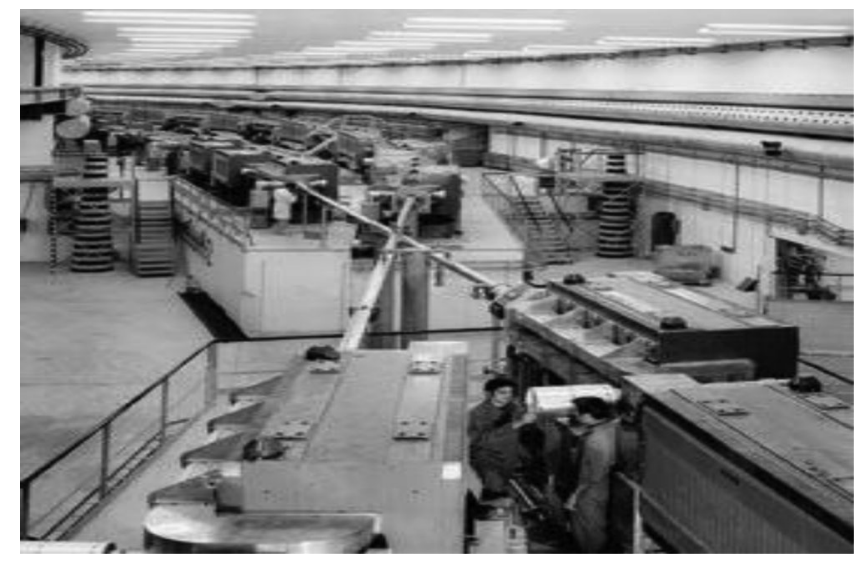

FIG. 5. Interaction point 5 in the ISR.

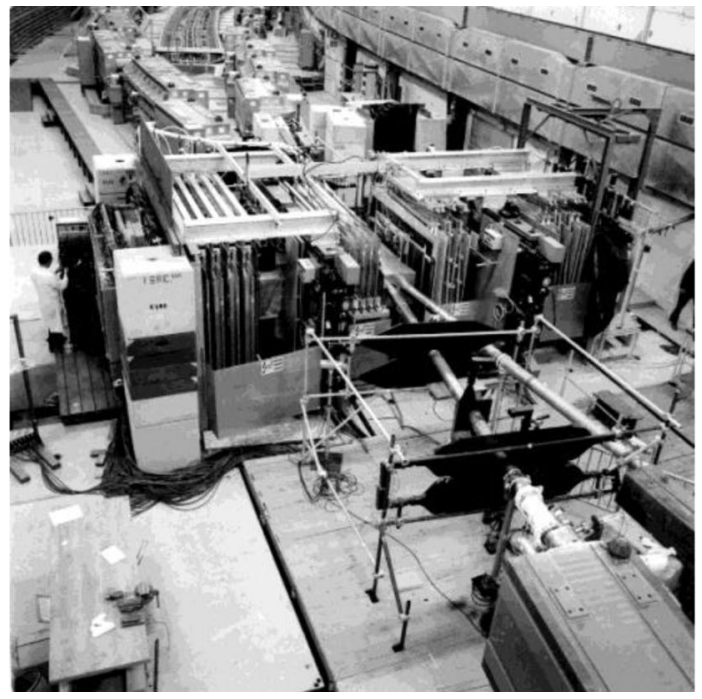

long straight sections in the interaction regions and the injection sections. The first proton-proton collisions took place in 1971 with beam momenta up to $26.5 \mathrm{GeV} / c$, which is the maximum momentum available from the PS injector.

I arrived at CERN in 1972, and the ISR had already shown that it could produce useful proton-proton collisions albeit with a rather low rate. My job was to be one of the "engineers in charge (EiC)" of the ISR, taking control of the operation during an eight-hour shift. The collider was run for 24 hours per day (three shifts) and, on a daily basis, each shift had one EiC and two operators.

At that time, I had no experience at all of working with accelerators. I was just writing up my Ph.D. thesis when I was hired at CERN and had a lot to learn. Being the person in charge of operating the ISR and providing physics to the experiments was a fantastic, fulfilling experience with an enormously fast learning curve. Everything was new and there was so much to learn. After about a year of experience, mistakes, expert training, and some self-training, I became proficient in tuning the collider performance to new record levels and gained a reputation as a good EiC. The schedule for the ISR was split between physics data taking and accelerator development, typically two days per week trying new techniques and equipment to improve the performance.

\section{Lack of diagnostics in ISR due to coasting beam}

High level, precise beam diagnostics are essential in a particle collider. They allow you to measure the number of particles in a beam, the beam dimensions and their position. In almost all accelerators the beams are formed into packets of protons (bunches). So, if you could observe the bunches at a specific point on the circumference of the accelerator, you would see a bunch go past then see nothing until the

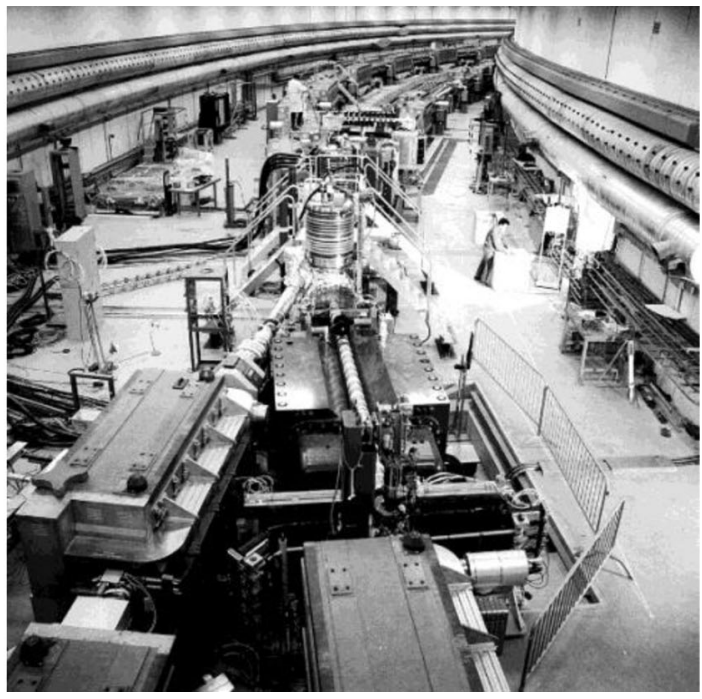

FIG. 6. Physics interaction points 1 (left) and 8 (right). 
next bunch passes. The bunches of protons are highly charged (protons are charged particles which allow you to manipulate them with electromagnetic fields). Nearly all beam diagnostics rely on the charged nature of the bunches to measure the properties of the beam. A charged bunch passes and induces an electromagnetic (EM) signal on the diagnostic device which decays after the passage of the bunch. The "induced" EM signal can be processed to evaluate many of the beam properties such as the number of protons, the position of the bunch in the vacuum chamber and the length of the bunch etc.

The ISR was a "storage" ring. This meant it took a batch of bunches from the injector PS, accelerated them to the storage aperture in the ISR and then "let them go." The bunches were "let go" by switching off the rf acceleration system to allow the rf system to capture and accelerate the next batch. This meant that the stored beam in the ISR was a "coasting" rather than a bunched beam. Hence none of the normal EM beam diagnostics devices could be used to measure the parameters of the coasting beam! This was a huge disadvantage for efficient operation for physics.

In the beginning there were only two devices which allowed the operators to "see" the coasting beam: a real time transverse profile monitor and the dc monitor. The transverse profile monitor used a very thin curtain of gas (sodium) which traversed the beam at a single azimuthal position on the circumference of each ring. The circulating protons caused ionization of the gas molecules which allowed us to use a camera to see the cross section of a beam as it passed through. The other key measurement was the total beam current monitor, which was an extremely precise current transformer which could measure bunched and unbunched beams.

\section{Stacking}

The accumulation of the very high currents in the ISR relied on a process called momentum "stacking." A stack was built by accumulating a few hundred PS beams across the large momentum aperture of the ISR. A single cycle involved rf capture of the PS 20 bunches at the injection momentum orbit of $-2 \%$ and accelerating this beam (by changing the rf frequency) to a momentum orbit of $+2 \%$. When the beam reached its required orbit, the rf was switched off and the beam debunched. The maximum single beam current ever achieved was 57 amperes (yes amperes!).

\section{Phase displacement acceleration}

Phase displacement occurs when an rf bucket traverses a debunched beam. The particles in the debunched beam travel around the unstable trajectories associated with the bucket (outside the separatrix, see Fig. 7). Traversing a debunched beam from high momentum to low momentum produces an increase in the average momentum of the debunched beam by an amount equal to the phase space

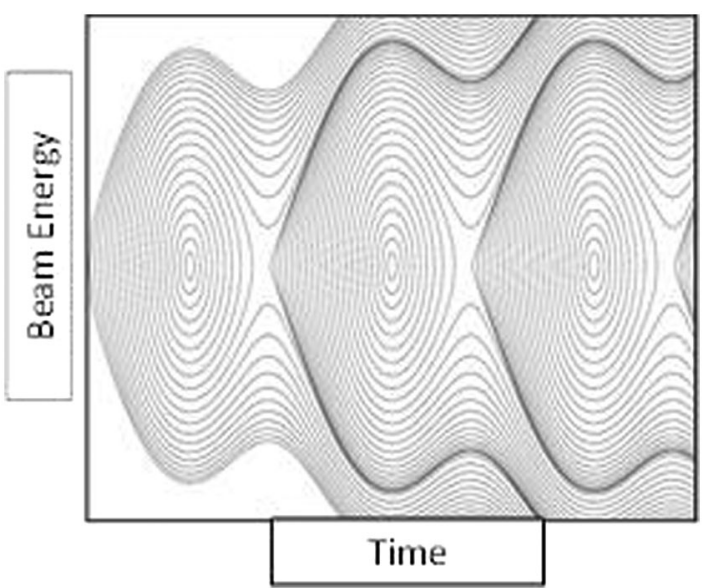

FIG. 7. Phase space trajectories of rf "buckets".

area of the phase displacing buckets. A good analogy is the release of droplets of mercury into a cylindrical container containing some water. The mercury droplets go from high energy to low energy and the water energy is increased by displacement.

Since the ISR circumference was larger than the PS, the maximum possible energy was also higher (31.4 compared to $26.6 \mathrm{GeV}$ ). In the never-ending quest for higher beam energies, it was decided to attempt to increase the energy of the accumulated beam in the ISR. However, the small ISR rf (16 kV maximum rf voltage) system could only capture a very small amount of a beam which had a 3\% momentum spread. So, in our relative ignorance of the problems (space charge, changing tunes, chromaticity, orbits, rf noise effects, absence of diagnostics, ...) we decided to attempt to phase displace high intensity stacks of protons. Initially the progress was very slow and frustrating, but after some better understanding and a few breakthroughs, $31.4 \mathrm{GeV}$ became the preferred high luminosity operational beam energy of the ISR.

\section{Working lines and space charge compensation}

The ISR had a working line (not a working point) in the horizontal-vertical tune diagram. The large tune spread was needed from the stability requirement from chromaticity and the large momentum spread needed for beam stacking. The minimum tune range of around 0.07 (see Fig. 8) created difficulties to find an area in the tune diagram which would allow the coasting beam to be free of low order nonlinear resonances. The working line drawn in Fig. 8 had the stacked beam between the 3rd and 5th order resonances but necessitated traversal of the bunched beam across the family of 5th order resonances. It was well known that the space charge tune shift caused a "sagging" of the working line, rather like loading a beam with heavy weights. This had two effects, resonances (in Fig. 8 the beam would reach the main coupling resonance), and beam instabilities caused by the reduction of the chromaticity for the low 


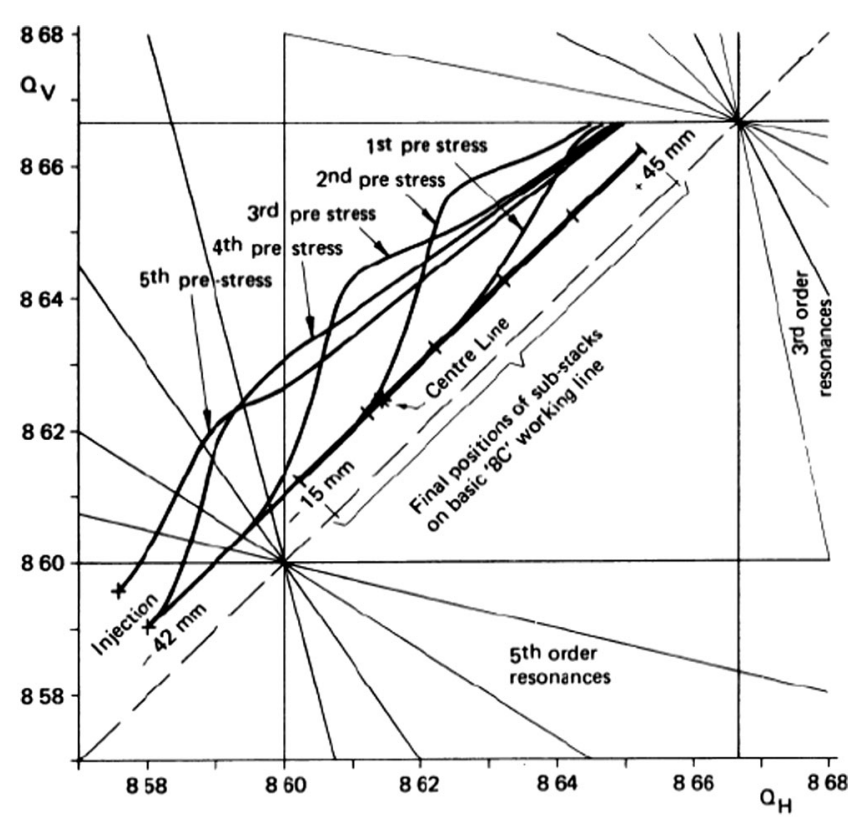

FIG. 8. Working line and space charge compensation.

momentum part of the stack. In order to be able to compensate the space charge effect we had (of course) to measure it. As previously stated, this is one of the major problems with unbunched beams; lack of diagnostics. A complicated system was developed which used beam transfer functions of empty buckets to measure the working line as a function of intensity. This system ultimately allowed measurements of the space charge tune shift which could be used for stepwise compensation during stacking. The measurement system was destructive to the beam (emittance) and never became robust enough to be used operationally. Figure 8 shows the procedure for space charge compensation while stacking [9]. The working line was "pre-stressed" for currents of 3 amperes and after the 3 amp increment of current had been stacked the next prestress was applied. Figure 8 shows the prestresses up to a total of 15 amps. This space charge compensation system took advantage of the great magnetic flexibility allowed by the combined function magnets and the inclusion of 24 pole face windings. The method also greatly stressed the capabilities of the controls system of the early seventies.

\section{Computer control of accelerators}

In order to accelerate high intensity "coasting" beams from 26 to $31 \mathrm{GeV} / c$, the computer control system of the ISR needed many upgrades and modifications. The acceleration by phase displacement involved around $200 \mathrm{rf}$ frequency sweeps through the beam. Each sweep increased the momentum by around $25 \mathrm{MeV} / c$ and necessitated incremental changes of the bending field so as to keep the beam in the center of the aperture. Due to the changing space charge effects, changes had also to be made to the tune and the chromaticity of the beams. The procedure

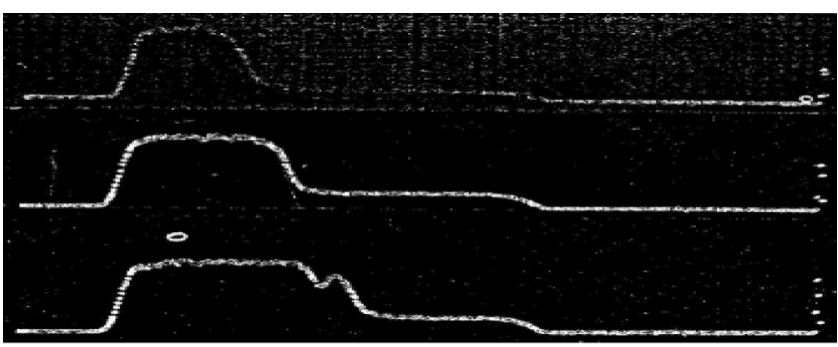

FIG. 9. Longitudinal Schottky scans.

developed involved many reference "break-points" as a function of energy and the acceleration between these break-points was done by interpolation. This same procedure was subsequently employed for the acceleration of beams in LEP and the LHC.

\section{Schottky scans}

The Schottky noise spectra [10] result from the discrete nature of the particles in the beam. A sensitive high frequency longitudinal pickup with some long-term signal processing using a spectrum analyzer could produce a signal proportional to the longitudinal phase space density of the debunched beam. Figure 9 shows one of the first Schottky scans taken operationally in the ISR. The three scans were taken at beam currents of 10,15 and 19.2 amperes. The horizontal axis is the longitudinal frequency and allows evaluation of the beam $\Delta \mathrm{p} / \mathrm{p}$.

Soon after discovering longitudinal Schottky scans, transverse pickups were used to measure the transverse Schottky scans which gave some information about the tune values in the stacked beams.

The operational use of these Schottky scans completely transformed the way of operating the ISR. On the long "stable-beams" fills, they were the only diagnostic available for observing the beam in a quantitative way (there was also the very useful sodium curtain which allowed visual inspection of the cross section of the beam). In the longitudinal plane the longitudinal density could be evaluated as a function of $\Delta \mathrm{p} / \mathrm{p}$ by incorporation of the value from the current meter. In addition, any "markers" on the stacks which could be identified in all planes would allow an evaluation of the location of this marker in tune space. The most usual markers for some time were the edges of the stack.

\section{Centering the accumulated beam in the aperture}

Injection to the ISR was performed by an injection kicker on a mechanically movable girder. When the stacking process was completed the girder was moved out so that the injection kicker was outside the aperture of the machine. This of course meant that, for maximum stacking aperture, the stack was situated on the "outside" of the beam aperture (see Fig. 10, left). Consequently, at the end of the stacking process, the stacked beams were not 


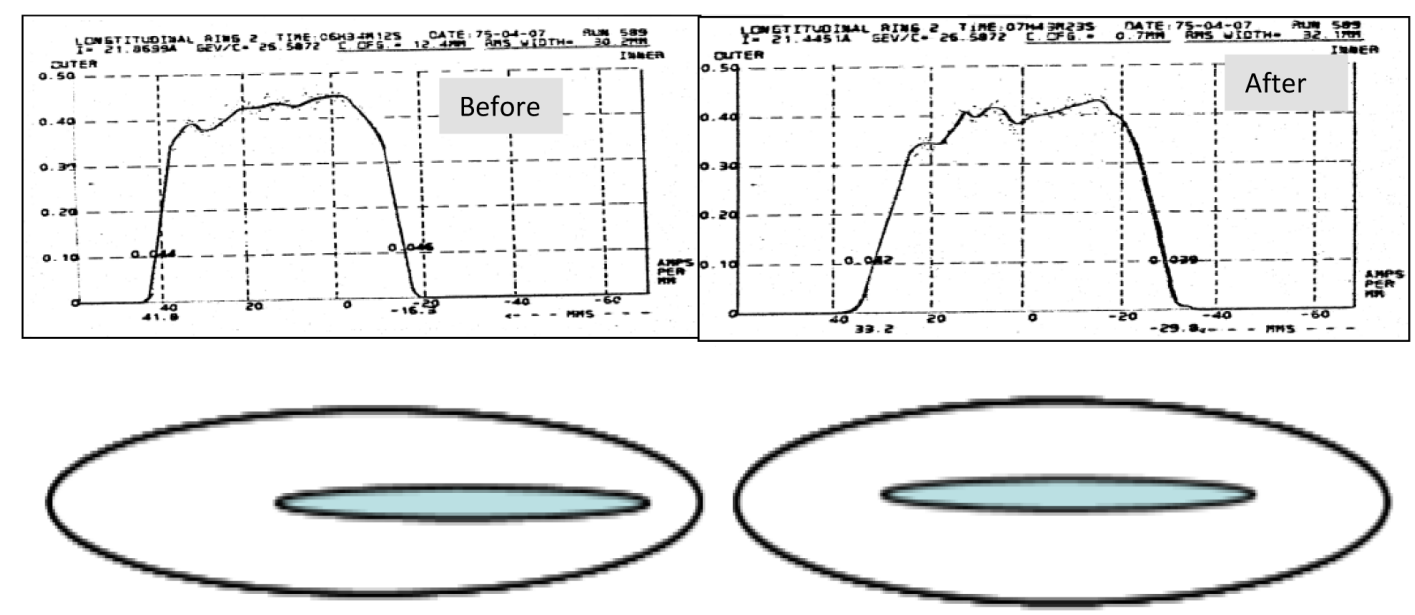

FIG. 10. Stack centering: before (left) and after (right).

centered in the aperture. This situation remained for several years and produced high background rates in the experiments due to the diffusion of particles from the "top" of the stack and scattering from the outer vacuum chamber wall. These high background rates reduced enormously the efficiency of the data accumulation in the experimental detectors. In 1975, the "background" in the ISR was the most serious operations problem which existed. On a typical physics run the EiC spent a lot of his time "scraping" the halos of the beams. These halos diffused from the top of the stacked beam and ended up as losses in the detectors.

I thought of a way to move the beam back into the center of the aperture by increasing the main bending field appropriately. This sounds trivial but the space charge compensation for the high current stack also had to be included and synchronized with the change of the bending field. I decided to try out this technique during one of the "graveyard" shifts. Control systems for accelerators in the mid-1970s were very primitive in comparison to today. There were several hundred power supplies whose values had to be changed in perfect synchronism. I calculated the changes to each power supply and loaded up the files to make a total movement of $8 \mathrm{~mm}$ of the total beam. It was very early in the morning by the time that I had prepared the necessary manipulations and files, and I was mentally exhausted. Since it was early morning, I knew that many of the bosses would be passing by the control room very soon. So, I had to try out the exercise before they came in, otherwise they would "advise" against such a risky procedure. I suspected that the most likely thing that would happen would be the abortion ("dump") of the total beam by the beam protection system, due to losses provoked by a miscalculation in the power supply settings.

I plucked up the courage to initiate the power converter changes which I had entered into the control system files. When the power converters started to move, I stared without blinking at the "Unser" current monitor [11] which could show changes as small as micro-amps (one millionth of an amp). I was absolutely amazed and shocked: the current in the Unser display started to increase! I could physically see (on the sodium curtain monitor) the huge number of protons moving inwards to the center of the aperture and the current kept on increasing. I later worked out why the "current" reading had increased: the beam was of course moving onto a smaller diameter closed orbit and was therefore making more turns per second: i.e. the rate of change of charge had increased because the time to make one revolution had diminished by a tiny amount. The high intensity beams had been displaced by $8 \mathrm{~mm}$ into the center of the ISR aperture. This procedure relied on the measurements made available with the Schottky scans (see Fig. 10

\begin{tabular}{|c|c|c|c|c|c|c|}
\hline \multirow{2}{*}{ Run } & \multirow{2}{*}{$\begin{array}{l}\mathrm{I}_{1} \\
\mathrm{amps}\end{array}$} & $\begin{array}{c}\mathrm{I}_{2} \\
\mathrm{amps}\end{array}$ & $\begin{array}{c}\frac{2}{\mathrm{~d} i} \mathrm{at} \\
\mathrm{at}\end{array}$ & $\begin{array}{c}\frac{-\mathrm{di}}{\mathrm{at}} 2 \\
\mathrm{ppm} / \mathrm{min}\end{array}$ & $\begin{array}{c}\frac{\mathrm{di}}{\mathrm{at}} 1 \\
\mathrm{ppm} / \mathrm{min}\end{array}$ & $\begin{array}{c}\frac{\mathrm{di}}{\mathrm{at}} 2 \\
\mathrm{ppm} / \mathrm{min}\end{array}$ \\
\hline 593 & 24.0 & 24.0 & 60 & 10 & 0.8 & 0.8 \\
594 & 24.0 & 24.0 & 10 & 10 & 0.8 & 0.8 \\
\hline
\end{tabular}

FIG. 11. Beam decay rates before and after beam centering. 


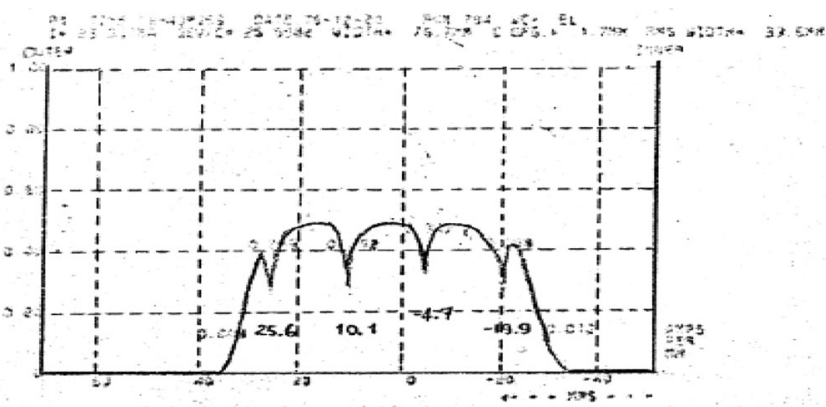

FIG. 12. Longitudinal Schottky with markers inserted by phase displacement.

which shows the scans before and after centering and the real beam position in the vacuum chamber).

When the procedure was finished, the telephones started ringing (yes, we did communicate with the experiments by telephone in the mid-1970s) and the response was fantastic. Unanimously all experiments declared that they had never seen such excellent background conditions. In addition, the Unser monitor, which also measures the decay rate of the beam current now gave a reading of 0.8 parts per million per minute (Fig. 11), corresponding to a beam lifetime of more than two years. Before the centering, the loss rate was at least 20 times higher (see Fig. 11). This procedure became standard procedure for all future "fills."

\section{Inserting markers in the stack}

As previously explained, complete traversal of the stack by empty rf buckets causes a change in the average momentum of the whole beam. It eventually became clear to me that partial traversal would change the momentum of that part of the stack that has been traversed. This was a simple procedure, the rf could be programmed to go from low momentum (outside the stack) to a momentum inside the stack, by simply programming the frequency swing. In this case a small reduction in the average momentum of the traversed part of the stack occurred, leaving a "marker" (lower density) at the $\Delta \mathrm{p} / \mathrm{p}$ where the rf traversing bucket stopped.

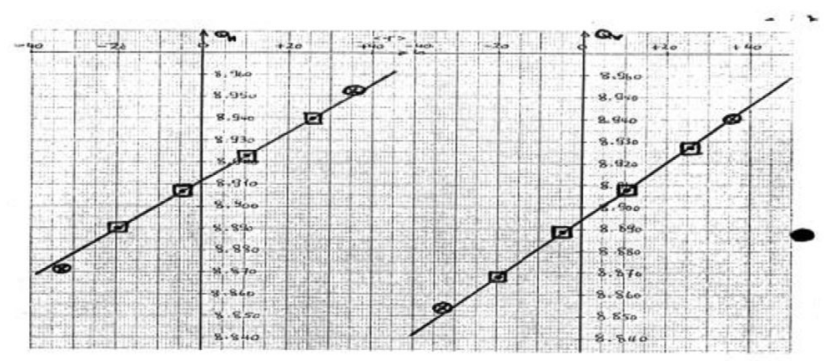

FIG. 14. Resulting measurement of the working line.

Figure 12 shows a longitudinal Schottky which had four markers inserted in this way. The markers are very clear and corresponded precisely to the programmed frequency of the rf stop. Of course, to be of any diagnostic use these markers must also be seen in the transverse plane.

Figure 13 shows the corresponding scans for the horizontal and vertical planes for the " $(n-q) \cdot f_{\text {rev }}$ " modes of oscillation. The corresponding markers are clearly visible in the transverse plane.

The combination of these measurements allowed plotting of the working line (see Fig. 14 for this particular case) in a nondestructive way and the markers lasted throughout the many hours of the physics runs.

\section{Working close to the integer}

In the early days (lower intensity, hence lower chromaticity) the working line was situated just above the half integer (8.5). In the latter, higher intensity days, when more tune spread was needed for the stability of the high intensity stacks, we were forced to operate just below the integer resonance (9.0) since this is the most resonance free area on the tune diagram. The top of the stack was situated at a horizontal tune value of 8.955 , just 0.045 distant from the integer. Initial operation at these new tune values was very problematic (orbit stability, transverse stability etc.), but with time all these known problems were solved. However, there was an effect unknown at the time, which caused a massive increase in the beam emittance over the top portion of the stacks. The sodium

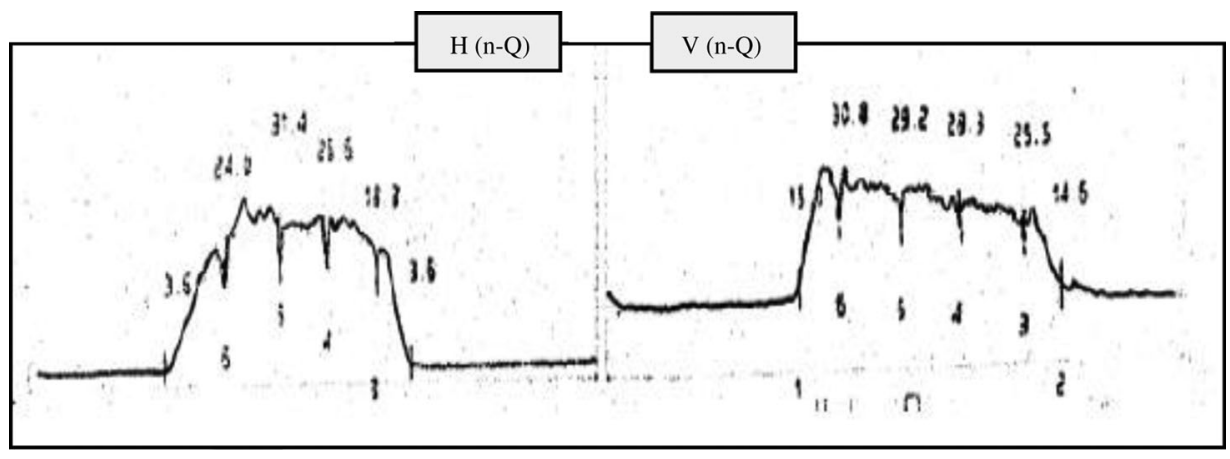

FIG. 13. Transverse Schottky with same markers apparent. 


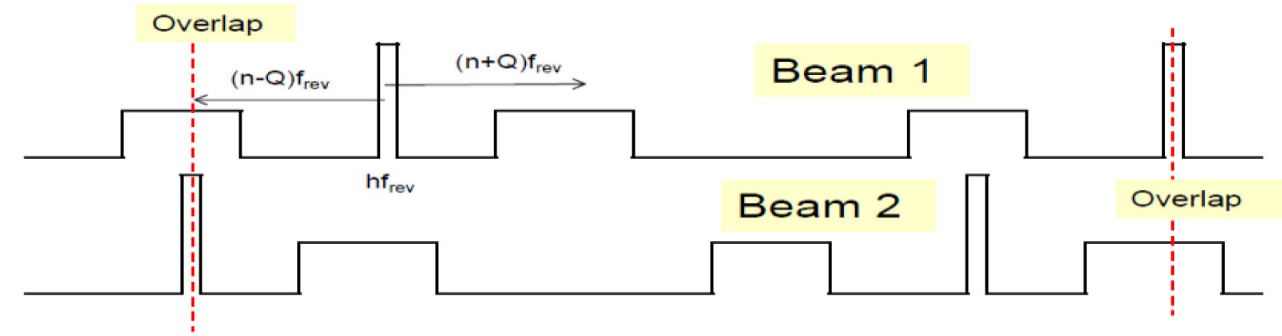

FIG. 15. Transverse frequency overlap.

curtain showed transverse cross sections of the beams which resembled lacrosse sticks.

\section{Overlap knock-out resonances}

In the ISR we had to worry about four beams: two beams per ring. The bunched beam at injection and during acceleration and the debunched already accumulated beam.

For beams that have significantly different revolution frequencies (caused by different $\Delta \mathrm{p} / \mathrm{p}$ or different charge/ mass ratios), overlap knock out (OLKO) was discovered to be an effect where the longitudinal harmonics of the bunch spectrum have components which are equal ("overlap") to the transverse betatron frequencies and thereby, by some form of coupling, can excite the beam at its transverse resonant frequency ("rf knock-out") as shown in Fig. 15.

The OLKO resonance condition, Q vs $\Delta \mathrm{p} / \mathrm{p}$ can easily be evaluated and is depicted in Fig. 16 for the various

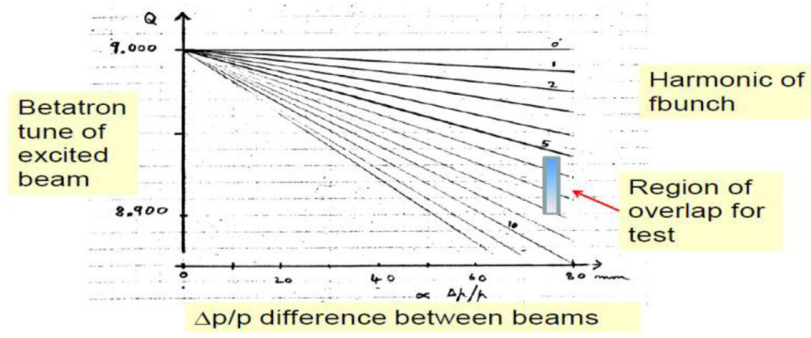

FIG. 16. The OLKO resonance conditions for the ISR.

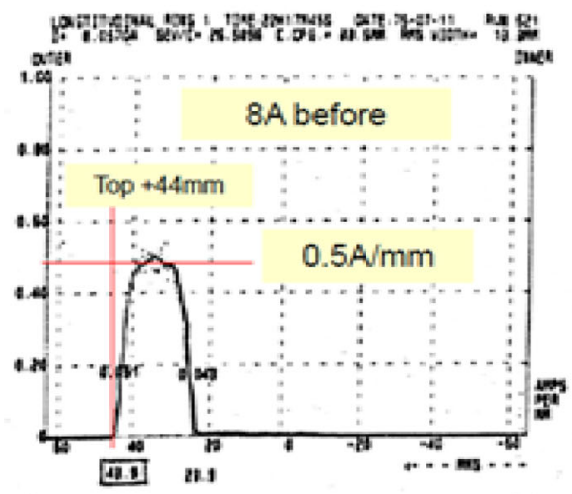

harmonics of the bunch frequency. Clearly this condition is much more easily met at lower harmonics of the bunch frequency when the transverse tunes approach the integer.

I performed a machine experiment to test the strength of this suspected effect. A beam of 8 amperes was accumulated over the tune space shown in Fig. 17 and collimated by scrapers (scatterers) so that any emittance increase would be recorded as beam losses (we could not measure directly the emittance). A bunched beam of $80 \mathrm{~mA}$ was injected in the other ring and allowed to circulate for 360 seconds. Figure 17 shows the longitudinal Schottky scans before and after the $360 \mathrm{~s}$ presence of the injected beam. The OLKO effect reduced the total current from 8 to $3 \mathrm{~A}$, the peak longitudinal density reduced from 0.5 to $0.26 \mathrm{~A} / \mathrm{mm}$ and the whole top part of the stack had been eroded. The beam-beam tune shift exercised by the $80 \mathrm{~mA}$ bunched beam was of the order of $10^{-6}$ !

The OLKO effect was studied extensively in the ISR and following many experiments, I proposed new procedures to allow operation very close to the integer. The cures used operationally were: (i) reduction of the higher harmonics of the bunch spectrum by bunch lengthening (lower $\mathrm{rf}$ voltage), and (ii) use of separations in the interaction regions so that the vector sum of beam-beam kicks over one turn is minimized.

In order to complete the study, tests were also done with colliding bunched beams with future accelerators in mind. In general, with bunched beams the resonance condition is not met if the rf frequencies of both beams are locked. For

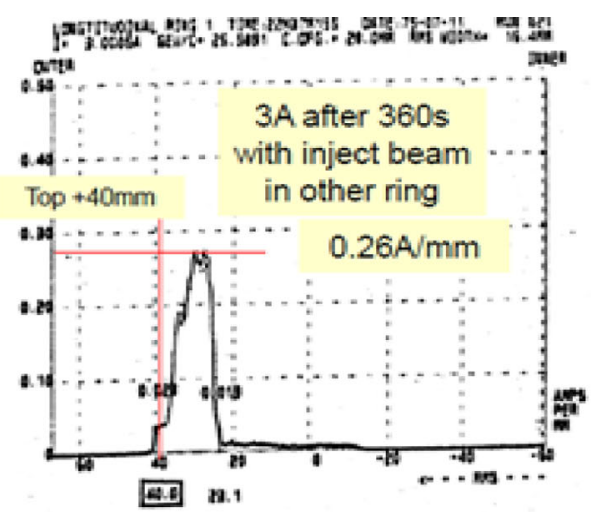

FIG. 17. Density profiles before and after OLKO. 


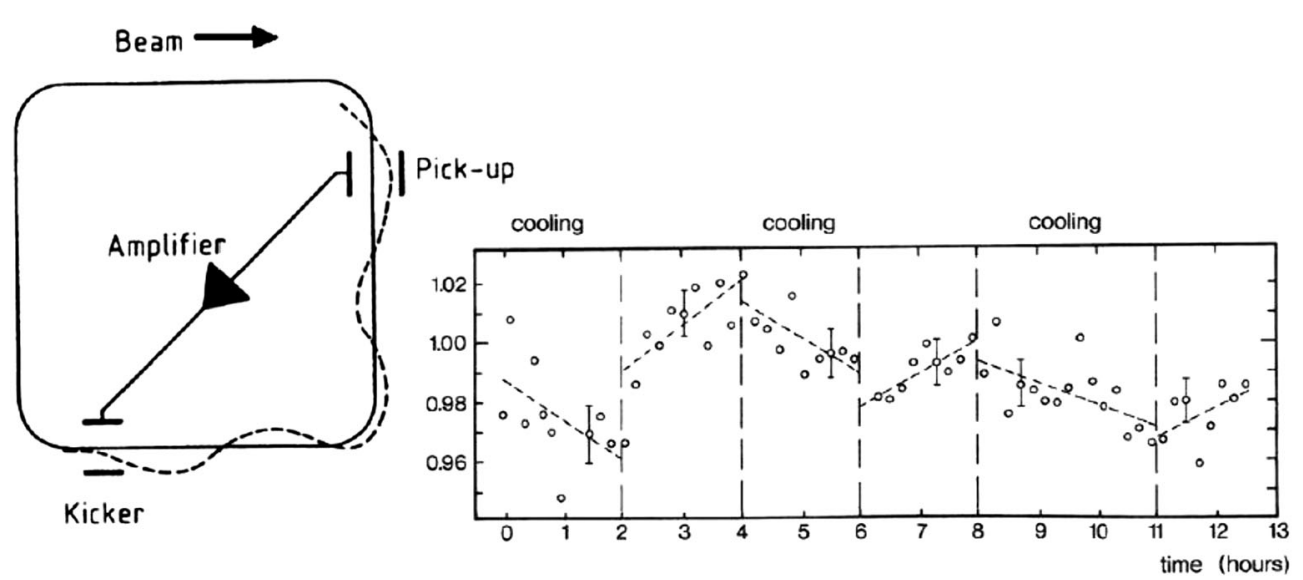

FIG. 18. First observation of stochastic cooling.

cases where frequency locking is not possible (e.g. resulting orbit is outside the vacuum chamber!) OLKO can be very destructive. This is particularly true for beams of different species and may cause operational difficulties for LHC colliding protons with lead ions.

\section{Stochastic cooling}

The first observations of the stochastic signals in the ISR (Schottky scans) immediately turned attention to the possibility of damping the oscillations of the particles (stochastic cooling). Significant effort in this direction was led by Wolfgang Schnell [12] following the initial idea by Simon Van Der Meer [13]. A stochastic cooling test system was built by Wolfgang Schnell's team as a demonstrator. The most sensitive detection of transverse beam size in the ISR was through the normalized luminosity measurement.

Figure 18 shows the results of the first ever conclusive observation of stochastic cooling (in an ISR machine physics experiment).

The normalized luminosity is shown over a 13-hour period with stochastic cooling turned on and off every few hours. The effect is small but very significant: stochastic cooling worked! Very soon afterwards a similar system was designed for the initial cooling experiment (ICE) with spectacular results as shown in Fig. 19.

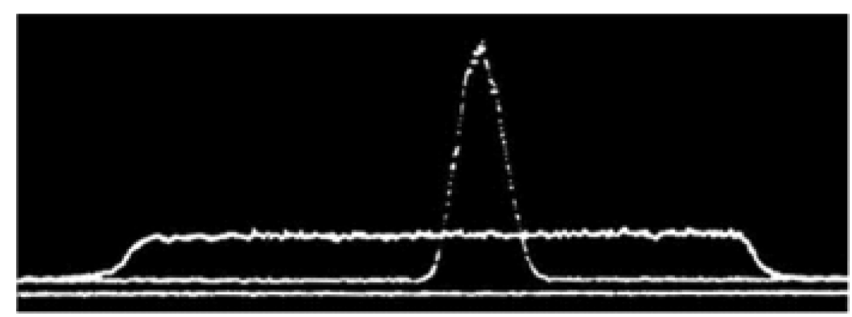

FIG. 19. Fast momentum cooling in ICE.

\section{Ultrahigh vacuum}

The ISR encountered many technological challenges but one of the most important, the ultrahigh vacuum (UHV), was imperative for a long beam lifetime. The stainless-steel vacuum chambers were baked in situ up to $300{ }^{\circ} \mathrm{C}$ in order to allow the very high beam intensities. The weakest vacuum sectors around the circumference were identified by "vacuum limit fills" where the intensity was increased to the value at which vacuum "runaway" occurred. Vacuum runaway resulted from the interaction of the high intensity beam of protons with the residual gas inside the vacuum chamber. This interaction caused the vacuum chamber walls to be bombarded by the generated particles or ions, and resulted in outgassing of the chamber walls with a concomitant increase in the vacuum pressure. The increased in vacuum pressure caused increased outgassing and a positive feedback mechanism. Consequently, when the circulating current reached a critical value, a vacuum instability occurred. This was an interesting challenge for the operations and rf teams as it meant that the stacking efficiency had to be maximized to produce the maximum longitudinal density in the stored "coasting" beam in order to reach the vacuum limit. I became an expert in achieving the highest beam currents and the vacuum group usually requested that I was the shift engineer during their vacuum experiment. The vacuum pressure and weak sectors were continuously improved during the life of the ISR.

The end result was an average pressure around $3 \times$ $10^{-12}$ Torr (see Fig. 20): one of the lowest pressures on earth or even in outer space. This superbly low vacuum allowed beam loss rates (due to beam gas collisions) of typically around one part per million per minute during physics runs (beam lifetimes of more than two years!). Beams of experiment quality could last 40 to $50 \mathrm{~h}$. Maximum intensities of up to $57 \mathrm{~A}$ were stored per ring with 30-40 A as typical values for experiments.

The maximum beam momentum was $31.4 \mathrm{GeV} / c$. With dc proton currents up to $40 \mathrm{~A}$ (single beam up to $57 \mathrm{~A}$ !) the 


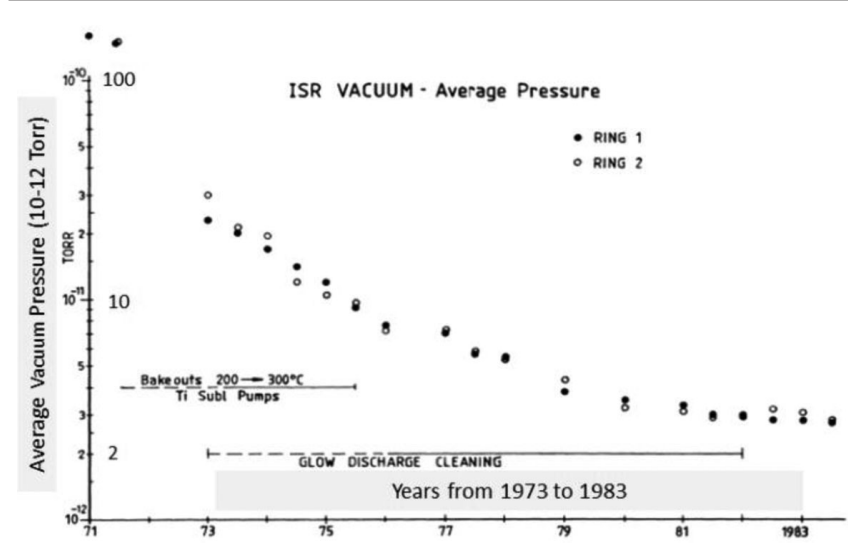

FIG. 20. Time evolution of the ISR vacuum pressure.

ISR reached a luminosity of $1.4 \times 10^{32} \mathrm{~cm}^{-2} \mathrm{~s}^{-1}$ in the superconducting low-beta insertion, a factor 35 above the design luminosity. It was a pioneer of accelerator technology.

The ISR operated for physics experiments from 1971 to 1983. It was decommissioned in 1984.

\section{An interesting experiment: Deuterons and protons in phase space}

As previously described, stacking in the ISR was performed in the longitudinal phase plane. Since deuterons and protons have different revolution frequencies, they occupy different positions in this phase plane. It should, therefore, be theoretically possible to stack protons and deuterons in the same real space since the stacks will be separated in phase space. As a machine experiment this was attempted for the first time in 1976, on a deuteron stack of 3.3 Amps which was left circulating while the parameters for proton acceleration were set up. A pulse of protons (0.15 Amps) was then injected onto the same vacuum chamber and accelerated into the "middle" of the deuteron stack. The beam profiles shown in Fig. 21 are obtained by the sodium curtain monitor. Figure 21 shows the position in real space of the proton beam with respect to the deuteron beam. The longitudinal Schottky scan of Fig. 22 shows

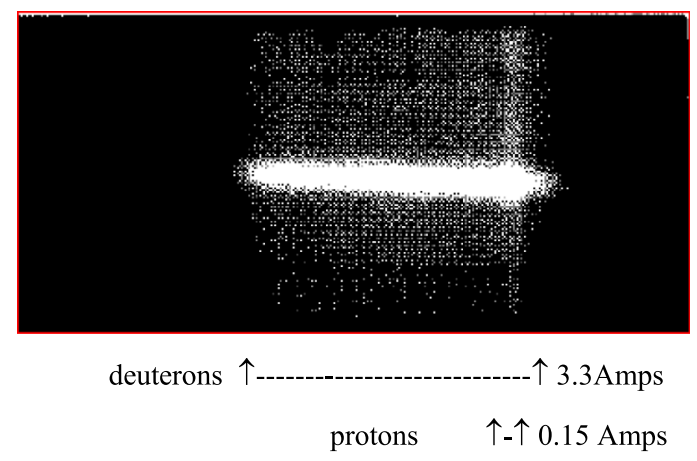

FIG. 21. Photograph taken from the gas curtain showing beams of deuterons and protons circulating in the same ISR ring.

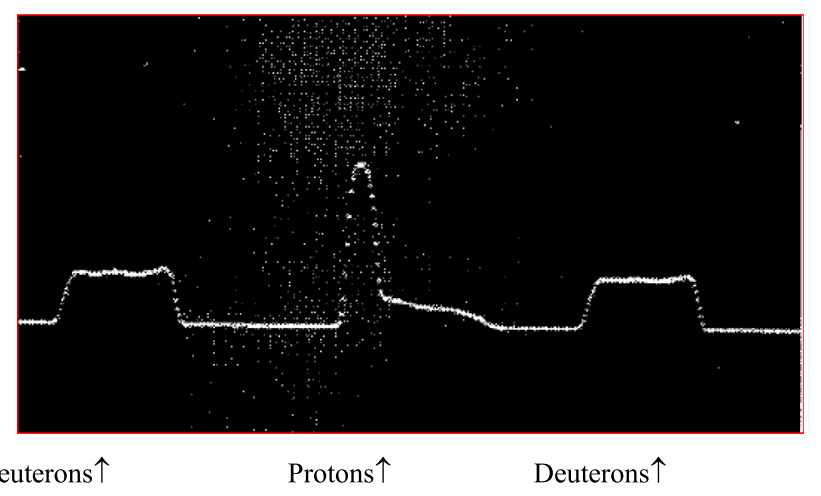

FIG. 22. Longitudinal Schottky scan showing deuterons and protons in the same vacuum chamber but occupying different frequencies in phase space.

quite clearly the separation (in phase space) of the beams of protons and deuterons.

The beam of protons was then traversed across its frequency range by empty rf buckets (phase displacement) so as to decelerate the beam. The frequency range was chosen so as to traverse the longitudinal frequency of the protons without going close to the deuteron frequencies. In this way, the protons were displaced in real space without influencing the position of the deuterons. The protons were finally decelerated in this way to a low momentum beam scraper, leaving once again only deuterons circulating in the ring.

\section{The legacy of the ISR}

Although the ISR has never been recognized as a discovery collider it is recognized as one of the most pioneering accelerator projects ever undertaken. Since it was the first pp collider, it allowed the production of a multitude of answers to long-standing questions as well as exciting new ideas for the future of particle colliders. It was also a fantastic training ground for accelerator physicists, and I was privileged to "earn my spurs" on this incredible project. The list of lessons learned and the future legacy of the ISR is long. Here I make a list of what I considered to be two of the most important.

How to build collider detectors.-I believe that the reduced fundamental physics output from the ISR was a direct result of the fact that the experimenters at the time did not know what type of detector should be used in a protonproton collider. The experiments were democratized, some looking at small angle events some looking at high transverse momentum events etc. All colliders following the ISR designed " $4 \pi$ "detectors to measure as many as possible of the simultaneous events resulting from the collisions. An immediate consequence of this was the UA1 and UA2 detectors for the SPP̄S.

Schottky noise and stochastic cooling.-Schottky noise diagnostics were first discovered and used in the ISR. The observation of these Schottky signals indicated that 
stochastic cooling could be possible. The first ever demonstration of stochastic cooling was performed in an iconic experiment in the ISR [12], where the collision rate (luminosity) was used as a measure of the transverse beam size. The direction and rate of change of the luminosity was clearly controlled by switching on and off the stochastic cooling electronics.

The long list of contributions made by the ISR.Although the two previously mentioned had, in my opinion, the greatest impact, the complete list of contributions to accelerator physics, operations, engineering is long and impressive. Many of these have been successfully implemented by more modern accelerators. In an arbitrary order of importance or priority the legacies left from the ISR are [14]: (i) a fantastic training ground for accelerator physicists and engineers, (ii) safe operation of beams with high destructive stored energy, (iii) background control by collimation, (iv) absolute luminosity calibration using "Van der Meer scans," (v) longitudinal phase space stacking of protons, (vi) production and maintenance of ultrahigh vacuum in the presence of high intensity beams (clearing electrodes, electron cloud, etc.), (vii) space charge tune compensation, (viii) impact of nonlinear resonances driven by machine imperfections, (ix) pulsed beam-beam effects (overlap knock-out resonances), (x) instabilities and impedance, (xi) phase displacement acceleration and deceleration, (xii) proton-antiproton collisions (demonstrated for the first time), (xiii) high precision, low noise, power converters, (xiv) low beta insertions, (xv) computer control of acceleration in colliders, (xvi) optics measurements and corrections, and (xvii) stacking and phase displacement of different particle species, protons, antiprotons, alfas, deuterons etc.

Insertion: Wolfgang Schnell, Simon van der Meer, Schottky scans and stochastic cooling.-One of my early memories was in the ISR control room when I was with Wolfgang Schnell, the group leader of the Radio Frequency group. We were looking at a spectrum analyzer when Wolfgang explained to me what we were observing on the spectrum analyzer.

It was clear that I had no idea what he was talking about, so he told me this intriguing story. He explained that during an ISR group leaders meeting in 1968, Simon van der Meer stated that beams of protons were NOT like an incompressible fluid (because the beams consisted of a very large number of individual protons) and were therefore not subjected to Liouville's theorem. This meant in laymen's terms that the size of the proton beams could in principle be reduced ("cooled") by a very high frequency feedback mechanism. What we were looking at on the spectrum analyzer was the proof that you could see the frequency spectrum of the multitude of protons. Wolfgang had realized that if you could see the protons (Schottky scans) you could act on them and reduce the size of the beam. When Wolfgang first observed these signals, he immediately phoned Simon and told him of his observations. Simon was not impressed and told Wolfgang that he was far too busy (designing and building the power converters for the SPS) to write up his statement from 1968. Wolfgang insisted on the importance of this work during several additional phone calls. Then, to get Wolfgang "out of his hair," Simon wrote a short report (August 1972) [13] giving some rather vague details about "stochastic cooling." In the "Final Note" of this report he wrote:

"Final Note.-This work was done in 1968. The idea seemed too far fetched at the time to justify publication. However, the fluctuations upon which the system is based were experimentally observed recently. Although it may still be unlikely that useful damping could be achieved in practice, it seems useful now to present at least some quantitative estimation of the effect."

Stochastic cooling was demonstrated in the ISR less than two years later and was used for the SPP S collider which discovered the $\mathrm{W}$ and the $\mathrm{Z}$ bosons. Simon van der Meer shared the 1984 Nobel Prize with Carlo Rubbia. The 1972 internal report was the basis for the Nobel Prize! Van der Meer was one of the very few accelerator physicists ever to have won the Nobel Prize, the others were Cockcroft/ Walton and Lawrence. If Wolfgang had been "dishonest" he could easily have "forgotten" what Simon had said verbally in 1968 and pursued stochastic cooling under his own name. He would almost certainly have been awarded the Nobel Prize with Carlo Rubbia.

\section{The future of CERN after the ISR and the SPS}

In 1974, the ISR was in full operation with beam, and the SPS was approved and under construction. Due to the known long lead time for accelerator projects, CERN bosses knew that the search for future projects had to be launched. Several study groups were set up to evaluate the various options for the future of CERN. All options were colliders because of the quest for ever increasing collision energies. From 1974 to 1978, the following options were studied in detail and feasibility reports were produced. (i) CHEEP: $27 \mathrm{GeV}$ electrons in an additional ring, colliding with $270 \mathrm{GeV}$ protons in the SPS, (ii) LSR/ SISR: pp collider with $400 \mathrm{GeV}$ per beam, (iii) MISR: $60 \mathrm{GeV}$ protons in a storage ring built from ISR magnets to collide with SPS protons, (iv) SCISR: two new superconducting rings for pp collisions with $120 \mathrm{GeV}$ per beam in the ISR, and acceleration by phase displacement, (v) proton-antiproton collisions in the SPS requiring the construction of an antiproton source, and (vi) large electron-positron (LEP) ring in a new tunnel.

In 1978, the decision was made for a proton-antiproton collider in the SPS as the medium-term project, and for LEP as the future long-term flagship, with the hope that it would be capable of discovering the Higgs boson. 
My first encounter with future "super proton colliders" was in 1977 when CERN was studying these possibilities for the future. I was nominated to contribute to the LSR/ SISR and the SCISR proposals. In the SCISR proposal, acceleration from 26 to $120 \mathrm{GeV}$ was proposed by phase displacement acceleration using the same tunnel and infrastructure of the ISR. During 1977 we produced three reports: (i) preliminary study of a superconducting conversion of the ISR (CERN-ISR/77-6), (ii) study of phase displacement to $120 \mathrm{GeV} / c$ (CERN-ISR/77-10), and in December 1977, and (iii) preliminary design study of Superconducting Conversion of the Intersecting Storage Rings, CERN 77-20.

The following year, I was invited to participate in the ISABELLE 1978 Summer Workshop at the Brookhaven National Laboratory, Upton, New York.

Insertion: Isabelle and RHIC.-The Isabelle project was recommended in 1974 by the U.S. High Energy Physics Advisory Panel to be built at Brookhaven. It was to be a $200+200 \mathrm{GeV}$ proton-proton collider using superconducting magnets. The project had been approved before development of the magnet technology had been completed. Construction began in 1978.

In July 1983, following the discovery of the $\mathrm{W}$ and $\mathrm{Z}$ bosons at CERN (see below), the U.S. Department of Energy canceled the ISABELLE project after spending more than US\$200 million on it. However, parts of the tunnel, experimental hall and magnet infrastructure built for ISABELLE were salvaged and reused by the Relativistic Heavy Ion Collider (RHIC) which was approved in 1991 and began operation in 2000.

\section{B. LEP and the beam-beam effect}

In 1979, CERN initiated a design study on the proposed 27-kilometer LEP collider. At the time, few thought CERN would ever build such a huge and costly collider. The people in charge of the project were Wolfgang Schnell, Eberhard Keil and "Kees" Zilverscoon. Schnell had been overviewing my performance with the ISR and asked me to leave the operations group and come and join him in the radio-frequency group as part of the then small design team of LEP. After seven years of operations shift-work combined with extra shifts for machine studies, I was ready to return to working more sociable hours. On top of that the project was incredibly exciting, and a great opportunity since Schnell was one of the most renowned and respected accelerator physicists at CERN.

LEP was an electron-positron collider, hence the name. Electrons are negatively charged, and positrons positively charged. Every electron-positron collider ever built before LEP was plagued with the dreaded beam-beam effect. Following my experience in the ISR particularly on the overlap knock-out effect, I decided to study the beam-beam effect and its impact on the performance of LEP. This was a huge challenge as many much more experienced accelerator physicists had attempted this in the past and none had been very successful. I decided to develop a "Monte-Carlo" computer simulation of the particles circulating in the collider. This involved "tracking" a large number of particles for a large number of revolutions around the collider.

Simulations in the past had been severely limited by the available computing power. The computing power in CERN had been recently upgraded and I thought it was worth a try with the new computer installations. Most of the computing power was dedicated to the experiments both for analysis of results and for preparation for LEP data taking. However, I managed to convince the CERN decision makers of the importance of the work and was granted time on the newly commissioned CERN mainframes. I also took advantage of my good personal relationships with some of the Scottish computer scientists running the center. It took a long time to develop and debug the simulation code but eventually the results appeared to reproduce the results on experiments on previous smaller colliders.

Andrew Hutton was one of the LEP design team and he decided to leave CERN to go to Stanford, California. At Stanford the PEP collider was in operation and was also suffering from the beam-beam effect. Andrew asked for a copy of my code and took it with him to Stanford. He ran the code and used it to propose a new "working-point" for PEP. In a very short period of time, the performance of PEP was significantly improved at the new working point. This was a significant validation of the code and I received a precious thank-you email from the Director of the Stanford Accelerator Centre, Burt Richter, saying that "simulation is a great thing!" Burt shared the Nobel Prize in 1976 with Sam Ting for the discovery of the J/ $\Psi$ meson in 1974: a period now known as the November revolution. Sam Ting was at this time spokesperson for the L3 experiment in LEP (the high energy world is a small world).

Eventually the code was used extensively to determine working conditions and predict the performance of LEP [15].

\section{The birth of LEP}

From 1979 I worked under the leadership of Wolfgang Schnell in the LEP machine design team. The first important decision was the circumference of the tunnel, with four options on the table: a $30 \mathrm{~km}$ ring which went deep into the Jura mountains, a $22 \mathrm{~km}$ ring which avoided them entirely, and two variants with a length of $26.7 \mathrm{~km}$. The final decision was (as usual) a compromise: the $26.7 \mathrm{~km}$ option but with a reduced amount of tunneling in the Jura foothills. The project was approved on 30 October 1981 just 11 months after Herwig Schopper took over as CERN Director General (DG). When Schopper was nominated by the CERN Council, there was a lot of dissention with the Italian delegation who had also proposed a strong candidate. Before the nomination of 
Schopper, Schnell, who was the driving force behind the technical design of LEP, was a hot favorite to be named project leader of LEP. With the nomination of Schopper as the new DG, and the dissention of the Italian delegates it became evident that a second German could not take the position of LEP project leader. Schopper named Emilio Picasso as the new LEP project leader. Emilio was a highly respected experimental physicist but had little experience with accelerators. This situation was perfect for causing conflict within the LEP project. I believe that Emilio wanted some expert impartial technical advice on the accelerator technology, so he nominated Gunther Plass as his deputy and created a LEP Machine Advisory Committee (LMAC). He nominated a highly renowned accelerator physicist Gustav-Adolf Voss from DESY as the chairperson of the LMAC. Schnell and Voss did not see eye-to-eye on most subjects, so this nomination poured petrol on an already dangerous fire. I was nominated as the scientific secretary of the LMAC, so I was caught in the crossfire, totally loyal to my boss and mentor, Wolfgang but with great respect for the new project leader and for the chair of the LMAC.

\section{Insertion: 1983, a proton-proton collider in the LEP tunnel: LEP note 440}

During the design phase of LEP, Wolfgang proposed the publication of a new series called "LEP notes." A LEP note could be published without any authorization from the higher powers in the organization. He thought that this was a good way to promote free thinking and inventiveness during the LEP design phase.

By mid-1981, the first $\mathrm{p} \overline{\mathrm{p}}$ collisions were observed in the SPP̄S at CERN. It was now very clear that ISABELLE was obsolete, and that the USA wanted a much higher energy collider.

Pressure was now building up among U.S. top physicists for endorsement of the super collider SSC. A series of meetings were organized in the U.S. during 1982 and 1983 to promote the SSC.

By chance I was invited to represent CERN at one of these meetings since none of the more senior accelerator staff were available. In addition, I had experience with the ISR, ISABELLE, and future CERN proton-proton collider proposals.

I was very much aware of the importance of these supercollider meetings and devoted a lot of time preparing for the meeting. I had many discussions and benefitted enormously from discussions with Wolfgang, we were both ex-ISR and leaned heavily on that experience. As a result of my participation in this U.S. discussion on future proton colliders, I became better known to the U.S. Department of Energy as well as the SSC proponents. During these discussions in the U.S., I began to think about the $27 \mathrm{~km}$ tunnel we would have in Geneva and what sort of proton collider we could imagine installing there. When I returned to Geneva, I told Wolfgang of my preliminary calculations on a future CERN proton collider.

Wolfgang then suggested that we augment and document this work in a LEP note. So, together we wrote LEP note 440 entitled "Preliminary performance estimates for a LEP proton collider."

LEP Note 440 was published in April 1983, and was a short, 16-page report that provided estimates for the design of a proton collider in the LEP tunnel and was the first document to bring all the ideas together. It raised many of the points that were subsequently part of the LHC design: $8 \mathrm{TeV}$ per beam, beam-beam limitation (arguing the case for a twinring accelerator), twin-bore magnets and the need for magnet development, problems with pileup (multiple collisions per bunch crossing) and impedance limitations.

When the copies of the note were ready for distribution, Wolfgang insisted on transporting some 400 copies or so to the CERN "courier" service himself in his own car. He told me that he did so in order to be sure and certain that there were no interventions to prevent the distribution! I really thought he had become paranoid, but history has proven him correct.

After these initial investigations, a dedicated CERN study group was established in late 1983 and the Lausanne workshop took place the following year, bringing experimental physicists together with accelerator experts to discuss the feasibility of the potential LHC. This was the beginning of a longer much more detailed technical design of the collider. Nevertheless, as Wolfgang predicted, there was never a citation of our work by the CERN study group.

However, Burt Richter (Nobel Laureate 1976) published the following in 2014 [16]:

"A Look Ahead to Beyond LHC-2014.

In the early 1980s the U.S. was beginning to develop the ideas for what became known as the Superconducting Super Collider (SSC), a 40-TeV center-of-mass superconducting $\mathrm{p}$-p colliding-beam machine ....

The first mention I know of what became the LHC is in an internal report from the LEP group (LEP note 440, 1983) by Stephen Myers and Wolfgang Schnell about putting a superconducting p-p collider in the LEP tunnel after the LEP e + e- collider had done its job....

The LEP tunnel was only roughly one-third the circumference of the SSC tunnel, but that could be partly made up for with higher field superconducting magnets (eventually the LHC magnets ran at about 30\% higher field than the design field of the SSC)....

The Myers/Schnell paper started informal discussions at CERN that became more serious when the SSC was initially approved by the U.S. Congress, and turned into a major design effort when the SSC was canceled by the U.S. Congress in 1993...."

LEP Note 440 was published on 11th April 1983 and has been available as a CERN official published document ever since and is still available from the CERN archives. 
In Wolfgang's obituary in 2006, reference was also made to the LEP note (see later).

We continued, and wrote more "LEP" notes $(450,460$, and 470) on the parameters for other subsystems for the LHC, and then abandoned this subject and went back to our real job, the design of LEP. However, over the next years, I did manage to keep in contact with future proton collider proposals mostly due to invitations to participate in the DoE reviews of the SSC in 1986 and 1990, almost certainly due to the authorship of this note.

\section{The CERN SPS proton-antiproton collider}

\section{The importance of stochastic cooling}

In a collider, the production rate (luminosity) is inversely proportional to the "phase-space density" (the three-dimensional beam size) of the colliding beams i.e. small beam sizes are needed for high luminosity. The "natural" beam size of the antiproton beam to be used for the SPPיS was many orders of magnitude larger than that needed for a "discovery" luminosity. The only existing way to reduce the beam size was by stochastic cooling as had been demonstrated in the ISR. However, the stochastic cooling experiment in the ISR had demonstrated cooling in a single phase-space plane (vertical). For guaranteed success of the SPP̄S project, it was clear that cooling must be demonstrated in three dimensions simultaneously in order to produce a reasonable event rate.

In February the "Initial Cooling Experiment (ICE)" was approved. The goal of this ambitious experiment was to demonstrate simultaneous cooling in all three dimensions in a new test ring. The ICE ring was constructed and commissioned in record time and successfully demonstrated cooling in May 1978. In June 1978, the decision was made to go ahead with the proton-antiproton experiment in the SPS.

Following the success of the test ring ICE, the antiproton accumulator (AA) was built also in record time, from 1979 to 1980 . The AA storage ring (157 m circumference, $3.5 \mathrm{GeV} / c$ ), was located downstream of a target which was bombarded with $26 \mathrm{GeV} / c$ protons from the PS and equipped with powerful stochastic cooling devices.

\section{Conversion of the SPS to the SPPיS}

The SPS had been built as a proton synchrotron but not as a collider. The following upgrades were needed if the SPP̄S project was to be successfully operated as $\mathrm{p} \overline{\mathrm{p}}$ collider.

A new beam line was needed, to transfer the antiprotons from the PS to the SPS, and a new injection system for counterclockwise injection was added in the SPS.

The SPS had been built for an injection energy of $14 \mathrm{GeV} / c$. The proton transfer line, TT10, and the injection system had to be upgraded to $26 \mathrm{GeV} / c$.

To provide sufficient beam lifetime for stored beams, the vacuum system had to be improved by 2 orders of magnitude, from the design vacuum of the SPS (200 nTorr) to better than 2 nTorr.

To increase the luminosity, tight focusing low-beta insertions were needed in straight sections IV and 5 for the UA2 and UA1 experiments.

Beam diagnostics had to be improved to measure the beam parameters with the very low beam intensities, and new devices added, such as directional couplers for independent observation of protons and antiprotons.

To reduce the beam-beam effect, high voltage electrostatic deflectors were required to separate the beams, in 9 of the 12 crossing points.

The rf system had to be upgraded with reduced "rf noise".

New transfer lines to and from the AA and from the PS to the SPS (TT70) were required.

The first proton-antiproton collisions were recorded in the summer of 1981. The first physics run took place at the end of that year when $0.2 \mathrm{nb}^{-1}$ of integrated luminosity was produced.

In the early operational years, the luminosity was pretty low, but still high enough to measure the existence of the W and $\mathrm{Z}$ in the runs of 1982 and 1983. Following this important discovery, Carlo Rubbia and Simon van der Meer shared the Nobel Prize in 1984. The large $4 \pi$ detectors, which had been denied to the ISR, had been decisive in the discovery.

During successive years the performance of the SPPיS increased dramatically following the inclusion of the antiproton collector (AC) to shorten the cooling times and to obtain a higher antiproton density. The energy of the individual colliding beams, $273 \mathrm{GeV}$ between 1982 and 1985, was raised to $315 \mathrm{GeV}$ from 1987 to 1991 when the program was terminated. The SPPS project was a hugely ambitious and successful project for CERN.

\section{The legacy of the SPP̄S}

The SPP̄S was designed as a hadron collider operating with bunched beams. Before its commissioning there were a lot of worries if such a machine could ever work, or if beam-beam effects would destroy the beams. It has to be said that colliding bunched beams had been tested previously in the ISR to accelerate and collide bunched beams of low intensities at $31.4 \mathrm{GeV}$ per beam. The SPP̄S removed all doubt about these worries for all future proton colliders.

For SPP̄S operation, the orbits of the protons and antiprotons were separated by a "Pretzel" shape by means of electrostatic separators. A similar technique was applied later to LEP and allowed an increase in the luminosity

In addition, the civil-engineering experience gained with the digging of the large caverns in the molasse bedrock for the two experiments UA1 and UA2 was extremely useful for the civil engineering of LEP and later the experimental areas of the LHC. 


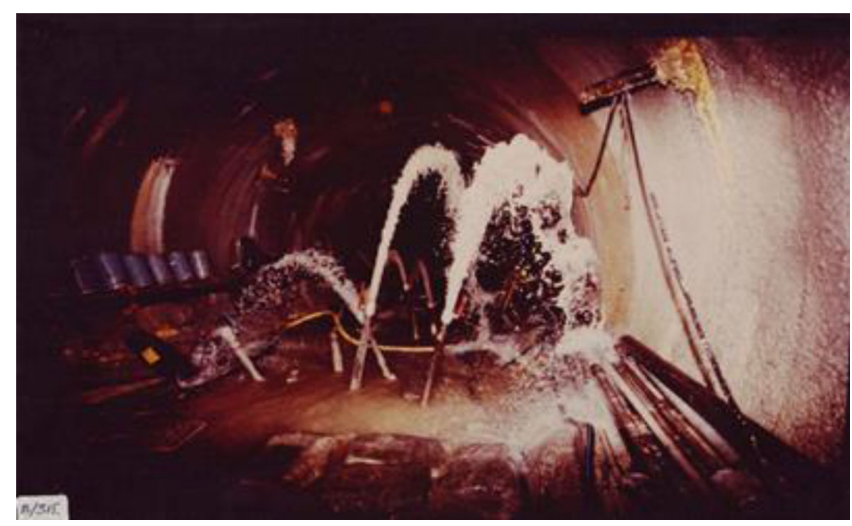

FIG. 23. Photo of water input to LEP tunnel.

\section{E. 1985 start of LEP construction}

The construction of the LEP tunnel started in 1985 following a standard public enquiry in France.

LEP's tunnel, the longest ever built, prior to the Channel Tunnel which links France and England, was carved by three tunnel-boring machines. Disaster struck just two kilometers into the three-kilometer stretch of tunnel in the foothills of the Jura. Underneath the mountain the rock had to be blasted because it was not suitable for the tunneling machines. Water burst into the tunnel and formed an underground river which took six months to eliminate (see Fig. 23 photo). This was a hard-earned lesson for the construction of future tunnels for colliders.

After many unsuccessful attempts to stop the highpressure water influx, a solution was found by June 1987. This allowed us to complete part of the tunnel and make it ready for the accelerator to be installed. However, the original smooth planning for construction and installation had now become much more complex.

\section{F. SSC DoE review (1986)}

I was invited by the Department of Energy to participate in the beam dynamics panel of the SSC review in 1986. The review was extremely well organized, very thorough and lasted a full week in Berkeley, California. While reading the preparatory documents and during the presentations, I became convinced that proper due diligence had not been carried out to a level which would ensure beam stability. Although this did NOT mean that I believed that the collider would not perform to specification, it meant that the risk level was higher that it should be.

When I presented the conclusions of the reviewers, there was a huge reaction. We discussed our findings late into the night and the designers of the SSC made every attempt to defend their design. The report from such reviews always has an "executive summary" and this is often the only part that high-level politicians read carefully. Initially my single paragraph describing the outcome of the beam dynamics review was considered to be much too direct and even negative. So, we engaged in the political wordsmithing of the single paragraph. Following a full day of backward and forward editing the document actually said absolutely nothing, which I believed was the intent. To illustrate that the document actually did not transfer any information I edited all the "is" to "is not," "are" to "are not" etc. and read the document aloud. There was a very mixed response to this exercise. Some of the more unfriendly scientists thought I should be thrown out and others thought it was hilarious. In the end I proposed a compromise text. By this time everyone was exhausted and fed up with the whole exercise and agreement was reached that the compromised version would be published in the executive summary. Part of the paragraph read [17]:

"...higher confidence in meeting performance goals would be obtained by further studies of magnet aperture requirements."

However, in the accelerator physics subpanel chapter of the same report (DOE-ER-0267), I concluded:

"At present the basic techniques employed to study the effects of magnetic field quality have not been developed to the extent required to conclude that the magnet designs for the SSC are conservative...one must hold open the possibility that the field qualities have to be improved... and for the dipole magnets an increase in aperture may be required...."

These two statements turned out to be real body blows to the SSC design team. Our accelerator physics subpanel also proposed that dynamic aperture tests be done at Fermi Lab, Chicago, in order to confirm the computer simulations. I knew how difficult it would be to get meaningful results from such experiments.

\section{Insertion: Dynamic aperture}

The "physical" aperture in a collider is defined by the surrounding beam enclosure; usually the dimensions of the inner wall of the vacuum chamber. The "dynamic" aperture is defined as the aperture inside which the beam (betatron) oscillations are stable in the long term. Particles which have amplitudes greater than the dynamic aperture will be driven to larger amplitudes and finally lost against the vacuum chamber wall. Clearly, for a very well-designed collider the dynamic aperture should be larger than the physical aperture. The beam instabilities which define the dynamic aperture are driven by higher order nonlinear magnetic fields e.g. in a bending magnet the fields which vary across the aperture of the magnet are unwanted higher field components.

Consequently, the design of the magnets in a collider is supercrucial to the success of the project. In particular it is important to minimize the nonlinear components of the wanted field. In addition, with superconducting magnets, these fields are more difficult to minimize than for room temperature magnets. In general, the smaller you make the magnet aperture, (for cost minimization) the more difficult 
it is to suppress higher order magnetic fields. The procedure for evaluation of the dynamic aperture in a proton collider is very technical and makes use of "beam simulations." In the 1980s and 1990s, the whole process was limited by the computing power available.

\section{G. 1989 LEP start-up}

LEP produced its first collisions on August 13, 1989, less than six years after ground was broken on September 13, 1983. The $27 \mathrm{~km}$ tunnel extends from the foothills of the Jura mountains to the Geneva airport and straddles the border between France and Switzerland. The $3.8 \mathrm{~m}$ diameter machine tunnel is buried at a depth varying between 50 and $175 \mathrm{~m}$.

The first job was the controversial octant test-the passage of a positron beam through the first eighth of the accelerator to be installed. It should be pointed out that there were many discussions by high ranking CERN personnel as to the value of performing an octant test. By July 1988, octant 8 (between the villages of FerneyVoltaire and Meyrin) was completely installed, the last part of the difficult excavation under the Jura having been finished just five months earlier. Soon after 23:55 on July 12 , 1988, four bunches of positrons made the first successful journey from Meyrin in Switzerland (point 1) to Sergy in France (point 2), a distance of $2.5 \mathrm{~km}$. It was an important moment and the culmination of several weeks of final hardware commissioning. Crucially, the beam measurements made on this first octant revealed a significant betatron coupling between the transverse planes (interference between the horizontal and vertical focusing of the beams) which was caused by a nickel layer (magnetic) inside the vacuum chamber used to bond radiation protecting lead $(\mathrm{Pb})$ to the aluminium $(\mathrm{Al})$ vacuum chamber. Discovering this defect one year ahead of the first circulating beam was invaluable.

The following months saw a huge effort to install equipment in the remaining $24 \mathrm{~km}$ of the tunnel-magnets, vacuum chambers, rf cavities, beam instrumentation, control systems, injection equipment, electrostatic separators, electrical cabling, water cooling, ventilation and all the rest. This was followed by the individual testing of 800 power converters, and connecting them to their corresponding magnets, while carefully ensuring the correct polarity. In parallel, the vacuum chambers were baked out at high temperature and leak tested. The rf units, which were located at interaction regions 2 and 6, were commissioned, and the cavities conditioned by powering them to the maximum power of $16 \mathrm{MW}$. Much of this had to be coordinated carefully to avoid conflicts between testing and installation work in the final sector, sector 3-4. At the same time a great deal of effort-with limited manpower - went into preparing the software needed to operate the collider, in close collaboration with the accelerator physicists and the machine operators.

In the late 1980s, control systems for accelerators were going through a major evolution which involved the use of PCs. LEP was caught up in the uncertainty and there were many differences of opinion as to which the correct control system for the LEP collider should be. Coming close to the planned first beam in July 1989, the control system was not ready, and a small team was created to allow the bare minimum controls to inject and ramp up the LEP energy. In any circular accelerator, the major objective is to circulate a beam. Only then can you measure critical parameters such as the "tune" values and orbit corrections. Therefore, I had two major concerns before start-up: Is the beam aperture clear of all obstacles, and are there any polarity errors in the connections of the many thousand magnetic elements? For LEP and later for the LHC I nominated a "Mr. Polarity" whose job was to check all polarities. This may sound trivial, but in the real case of tens of thousands of connections and naming conventions it is a huge task. (For example, QL10L1 refers to the tenth long quadrupole left of IP1. But what is left/right of the interaction point: is this looking from the inside of the circumference? Is it for the direction of the electron or the positron circulation: for cable connections, do you connect 1 to 1 or 1 to 2 etc.? Mr. Polarity took care of all these possible errors and misinterpretations). 


\section{Insertion: LEP and the WWW Tim Berners-Lee}

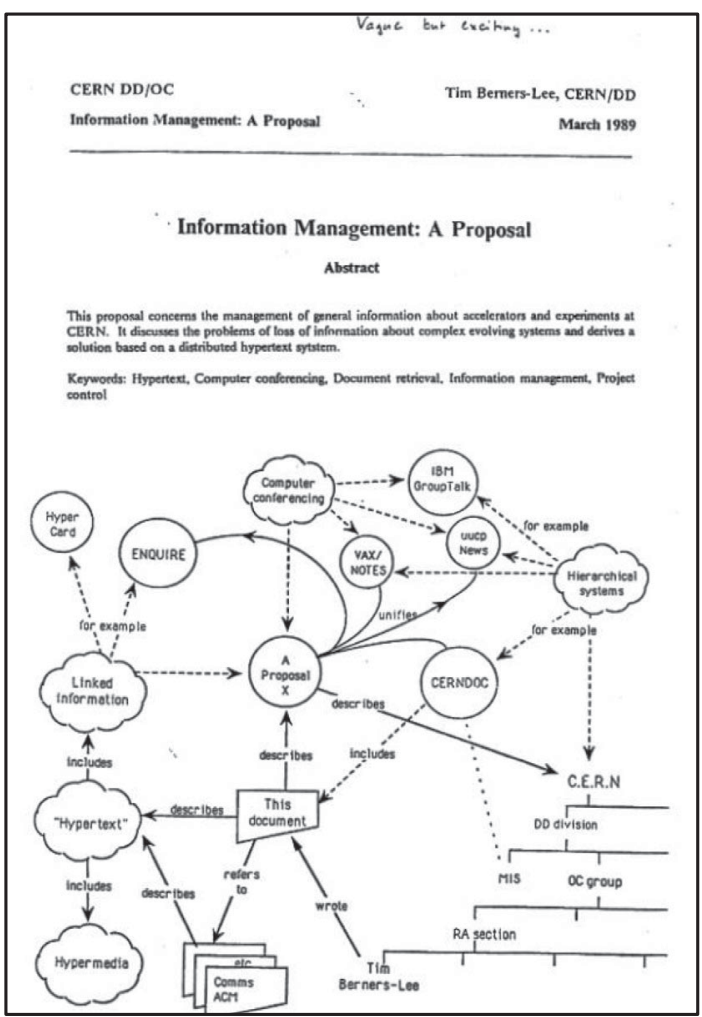

First circulating beam on 14 July 1989-The 200th anniversary of the storming of the Bastille.-At 23:45, on 14 July 1989, the aperture was indeed free of obstacles, and the beam made its first turn on our first attempt. Soon afterwards we managed to achieve a circulating beam, and we were ready to fine-tune the multitude of parameters needed to prepare beams for physics. This major milestone was accomplished with a minimalistic control system.

The goal for the first phase of LEP was to generate electron-positron collisions at a collision energy of around $90 \mathrm{GeV}$, equivalent to the mass of the $\mathrm{Z}$, the neutral carrier of the weak force. LEP was to be a true $\mathrm{Z}$ factory, delivering millions of Zs to perform precision tests on the Standard Model. To mass produce the Zs required beams not only of high energy, but also of high intensity. To deliver such beams required four major steps. The first was to accumulate the highest possible beam current at the injection energy $(20 \mathrm{GeV})$. A major operation in itself, it involved the purpose-built LEP Injection Linac and Electron-Positron Accumulator, the Proton Synchrotron, the Super Proton Synchrotron and, finally, transfer lines to inject electrons and positrons in opposite directions, which curved not only horizontally but also vertically as LEP and the SPS were at different heights. The second step was to ramp up the accumulated current to the energy of the $\mathrm{Z}$, with minimal losses. The third step was to "squeeze" (more strongly focus) the beams at the interaction points to improve the proton density and thereby the collision rates. The final step
Tim Berners-Lee was working in CERN in the computer division (DD). His task was to work on the distribution of the data coming from LEP to the universities all around the world.

LEP was due to circulate first beam in July 1989, Tim wrote the proposal (opposite) for his supervisor. The response from his supervisor was written on the proposal "vague but exciting".

This one-page note is the invention of the world-wide-web which has changed the way we live, work, communicate, shop etc. in today's world.

Tim's invention was designed to solve a data problem for LEP.

was to bring the beams into perfect "head-on" collisions using the electrostatic separators.

Following the highly successful first turn, we spent the next month preparing for the first physics run. Almost exactly one month after first beam, on August 14, we brought the beams into first collisions in the LEP collider. The next ten minutes seemed like an eternity since none of the four experiments-ALEPH, DELPHI, L3 and OPALreported any events. I was in the control room with Emilio Picasso and we were beginning to doubt that the beams were actually colliding when Aldo Michelini called from OPAL with the long-awaited comment: "We have the first Z0!"

I kept the copies of the first event and the signatures of the people (Carlo Rubbia, Emilio Picasso, Albert Hofmann, Wolfgang Schnell, John Poole, John Thresher, Carlo Wyss, Leslie Camilieri, plus some faded signatures) in the control room at the time (see photo in Fig. 24).

A period of machine studies followed, allowing big improvements to be made in the collider's performance.

\section{Insertion: The ZO bell}

During LEP's first "pilot" run for physics in August 1989, ALEPH and OPAL physicists connected the Z signal to a bell which sounded loudly and proudly on the arrival of the particle in their detectors. OPAL's bell was performing very well while that of ALEPH was silent (it turned out that 


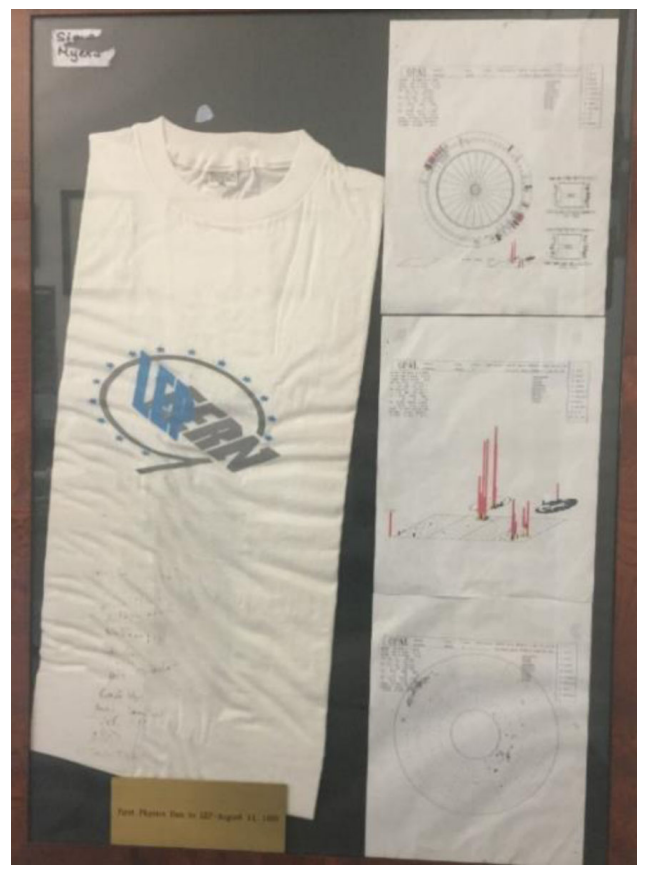

FIG. 24. LEP T shirt signed on the occasion of the first Zo.

they were waiting for the collimators to close before turning on the subdetectors). An hour later this error was corrected, and ALEPH's bell began to ring rapidly. As the luminosity rose, the bell became a bloody nuisance and was switched off.

On 20 September 1989, the first physics run began, with LEP's total energy tuned for five days to the mass peak for the $\mathrm{Z}$ and sufficient luminosity to generate a total of some $1400 \mathrm{Zs}$ in each experiment. A second period followed, this time with the energy scanned through the width of the $\mathrm{Z}$ at five different beam energies-at the peak and at \pm 1 and $\pm 2 \mathrm{GeV}$ from the peak. This allowed the four experiments to measure the width of the $\mathrm{Z}$ and so announce the first physics results, on 13 October, only three months after the final testing of the accelerator's components.

\section{H. SSC review in 1990}

The SSC had a proposed circumference 3 times larger than the existing tunnel (LEP) which was being proposed to house the Large Hadron Collider. Under these circumstances the collision energy of the SSC was much higher than anything which could be proposed for the LHC. The only way in which the CERN-LHC could be seen as competitive was by proposing a much higher rate of collisions ("luminosity"). The design luminosity for the LHC was purported to be 10 times higher than that of the SSC, but no one at CERN knew how or if this huge luminosity was at all achievable.

Following the 1986review (see previously), a site selection committee, which began in April 1987, was set up to evaluate proposed sites for the construction of the SSC.
In November 1988, the day after George Bush was elected to the presidency, it was announced that a site at Waxahachie, Texas, a town of 18,000 people about 25 miles southwest of Dallas, had been ranked outstanding on every major criterion by the site-selection committee. In addition, Texas had also promised to contribute one billion dollars to the project.

Following the announcement of the site selection, a second "site specific" review of the SSC was organized for July 1990.

I was invited, for a second time to chair the collider accelerator physics subpanel review. This was a total surprise as I thought I had made life-long enemies at the first review in 1986.

Before leaving for the U.S., I had a short meeting with the CERN Director General at the time, who suggested that I should convince the reviewers "that smaller is better." I interpreted this statement as a signal to do everything I could to promote the CERN LHC.

This was a very interesting period for colliders: LEP was now up and running and from a luminosity point of view was totally outperforming the Californian Stanford Linear Collider. On the other hand, SSC had received U.S. governmental approval which would surely preclude any form of approval for the LHC, since the world's physicists only needed one super collider. This did not look very assuring for the future of the world's leading high energy physics laboratory, CERN.

In September 1990, the review committee conclusions were reported [18].

"From June 25 to 30, 1990, an Office of Energy Research Review Committee (ERC) evaluated the technical feasibility, the estimated cost, the proposed construction schedule, and the management arrangements for the Superconducting Super Collider (SSC) as documented in Site-Specific Conceptual Design Report (SDR) and other materials prepared by the Superconducting Super Collider Laboratory (SSCL).”

The summary findings of the 1990 subpanel noted that the proposals from the previous review in 1986 had been followed: i.e.

"...Comparing the SCDR with the Conceptual Design Report (CDR) of 1986...Injection energy has been doubled to $2 \mathrm{TeV}$. The dipole magnet aperture increased from 40 to $50 \mathrm{~mm}$. A new 90-degree lattice and shorter cell length ...."

These technical changes had of course incurred substantial increases in the cost of the SSC. In the executive summary of the review, it was written that:

"...Escalating to as-spent dollars, using the escalation rates provided by the DOE and $\mathrm{OMB}$ and the funding profile developed by the SSCL, results in a Total Project Cost (TPC) calculated by the ERC of $\$ 8.4$ billion ...."

The $\$ 8.4$ billion was to be compared with the statement made in the 1986 Executive Summary (DOE-ER-0267): 
"The SSC CDR has documented the estimated cost for constructing an SSC facility at \$3.01 billion in FY 1986 dollars, ...

...The DRC finds that the SSC CDR cost estimate is credible and consistent with the scope of the project."

So, from 1986 to 1990, the SSC estimated cost had increased by $280 \%$ ! Quite recently a very interesting book has been published giving insight to the factors (political, scientific and financial) leading to the demise of the SSC.

In the collider accelerator physics subpanel which I was chairing, we noted that, although nearly all of the recommendations made in 1986 had been implemented, including the increase in the aperture of the bending (dipoles) magnets, those of the focusing (quadrupole) magnets remained at $4 \mathrm{cms}$. This meant that the vacuum aperture seen by the beam was repeatedly changing from 4 to $5 \mathrm{cms}$ and back to $4 \mathrm{cms}$ on each beam traversal of a dipolesquadrupoles pair of magnets. This provokes a certain increase in the "impedance" seen by the beam and can result in many different types of beam instabilities.

In the summary of the subpanel of the second review I wrote:

"...Consideration should be given to increasing the quadrupole aperture to $50 \mathrm{~mm} \mathrm{...} \mathrm{to} \mathrm{get} \mathrm{more} \mathrm{efficient}$ use of space and a smooth vacuum chamber with reduced impedance." This was another huge and costly proposed design change.

\section{LEP operations}

LEP was the highest energy electron-positron collider ever built, with levels of precision that remain unsurpassed in accelerator physics to this day. Here is the story of LEP from conception to its emotional final day.

A few minutes before midnight on a summer's evening in July 1989, a small group of us were crammed into a back room at CERN's Prévessin site in the French countryside.

\section{Insertion: CERN Control Center}

Indeed, our small team found ourselves running the largest accelerator ever built in what was basically a back room of the SPS Control Room at Prévessin. This was another lesson not to be repeated for the LHC and later resulted in the proposal for the CERN Control Centre.

The Large Electron Positron collider LEP at CERN was commissioned in 1989 and finished operation in November 2000. During this period, it was operated in different modes, with different optics, at different energies, and with excellent performance. In the end, LEP surpassed all design parameters. It has provided a large amount of data for the precision study of the standard model, first on the $\mathrm{Z}$ resonance, and then above the $\mathrm{W}$ pair threshold. Finally, with beam energies above $100 \mathrm{GeV}$, a tantalizing glimpse of what might have been the Higgs boson was observed.

LEP performance [19-22].-Performance at LEP naturally was divided into two regimes: at $45.6 \mathrm{GeV}$ per beam
TABLE II. Overview of LEP performance from 1989 to 2000. Note $\mathrm{E}_{b}$ is the energy per beam, $\mathrm{k}_{b}$ the number of bunches per beam and $\mathrm{L}$ is the peak luminosity.

\begin{tabular}{|c|c|c|c|c|c|}
\hline $\begin{array}{l}\text { Overv } \\
\int \mathcal{L} d t \\
\text { over } \\
\text { rent } 2 \\
10^{30} \mathrm{cr}\end{array}$ & $\begin{array}{l}\text { iew of } \mathrm{LE} \\
\text { is the lun } \\
\text { ach year } \\
k_{\mathrm{b}} I_{\mathrm{b}} \text {. Th } \\
\mathrm{n}^{-2} \mathrm{~s}^{-1} \text {. }\end{array}$ & $\begin{array}{l}\text { P performa } \\
\text { inosity inte } \\
\text { and } I_{\text {tot }} \text { is } \\
\text { luminosity }\end{array}$ & $\begin{array}{l}\text { ce from } \\
\text { rated } 1 \\
\text { the to } \\
\mathcal{L} \text { is gi }\end{array}$ & $\begin{array}{l}1989 \text { to } \\
\text { er expe } \\
\text { al bear } \\
\text { en in u }\end{array}$ & $\begin{array}{l}2000 . \\
\text { iment } \\
\text { cur- } \\
\text { its of }\end{array}$ \\
\hline Year & $\begin{array}{l}\int \mathcal{L} d t \\
\left(\mathrm{pb}^{-1}\right)\end{array}$ & $\begin{array}{l}E_{\mathrm{b}} \\
\left(\mathrm{GeV} / c^{2}\right)\end{array}$ & $k_{\mathrm{b}}$ & $\begin{array}{l}I_{\text {tot }} \\
(\mathrm{mA})\end{array}$ & $\bar{L}$ \\
\hline 1989 & 1.74 & 45.6 & 4 & 2.6 & 4.3 \\
\hline 1990 & 8.6 & 45.6 & 4 & 3.6 & 7 \\
\hline 1991 & 18.9 & 45.6 & 4 & 3.7 & 10 \\
\hline 1992 & 28.6 & 45.6 & $4 / 8$ & 5.0 & 11.5 \\
\hline 1993 & 40.0 & 45.6 & 8 & 5.5 & 19 \\
\hline 1994 & 64.5 & 45.6 & 8 & 5.5 & 23.1 \\
\hline 1995 & 46.1 & 45.6 & $8 / 12$ & 8.4 & 34.1 \\
\hline 1996 & 24.7 & $80.5-86$ & 4 & 4.2 & 35.6 \\
\hline 1997 & 73.4 & $90-92$ & 4 & 5.2 & 47.0 \\
\hline 1998 & 199.7 & 94.5 & 4 & 6.1 & 100 \\
\hline 1999 & 253 & $98-101$ & 4 & 6.2 & 100 \\
\hline 2000 & 233.4 & $102-104$ & 4 & 5.2 & 60 \\
\hline
\end{tabular}

running around the $\mathrm{Z}$ boson resonance and high energy running above the threshold for $\mathrm{W}$ pair production. A summary of the performance through the years is shown in Table II below.

In the regime on or around the $\mathrm{Z}$ resonance, performance was constrained by the beam-beam effect which limited the bunch currents that could be collided. The beam-beam effect blew up beam sizes and the beam-beam tune shift saturated at around 0.04. Optimization of the transverse beam sizes was limited by beam-beam driven effects such as flip-flop. The main breakthrough in performance at this energy was an increase in the number of bunches. First with the Pretzel scheme (eight bunches per beam) commissioned in 1992, and then with the bunch train scheme (up to 12 bunches per beam) used in 1995. The optics (phase

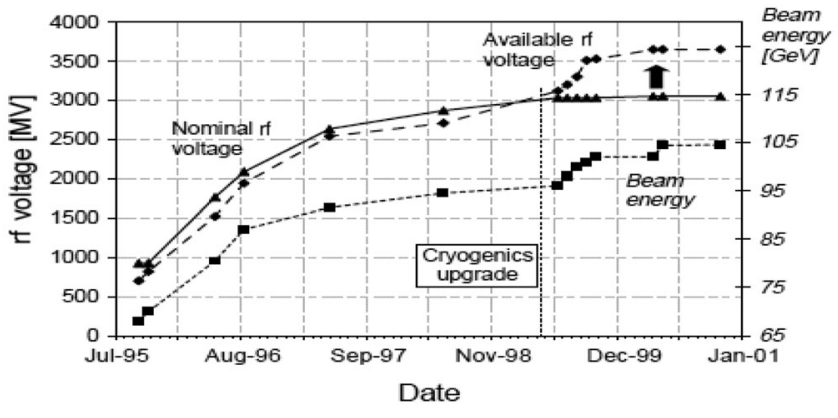

FIG. 25. Radio frequency voltage per turn over the years. 
TABLE III. LEP Performance parameters.

\begin{tabular}{|c|c|c|c|}
\hline Parameter & $\begin{array}{c}\begin{array}{c}\text { Design } \\
(55 / 25 \mathrm{GeV})\end{array}\end{array}$ & $\begin{array}{l}\text { Achieved } \\
(46 / 98 \mathrm{GeV})\end{array}$ & \multirow{8}{*}{$\begin{array}{l}\times 10 \\
\times 1.4 / 3.7\end{array}$} \\
\hline Bunch current & $0.75 \mathrm{~mA}$ & $1.00 \mathrm{~mA}$ & \\
\hline Total beam current & $6.0 \mathrm{~mA}$ & $8.4 / 6.2 \mathrm{~mA}$ & \\
\hline $\begin{array}{l}\text { Vertical beam-beam } \\
\text { parameter }\end{array}$ & 0.03 & $0.045 / 0.083$ & \\
\hline Emittance ratio & $4.0 \%$ & $0.4 \%$ & \\
\hline Maximum luminosity & $\begin{array}{c}16 / 27 \\
10^{30} \mathrm{~cm}^{-2} \mathrm{~s}^{-1}\end{array}$ & $\begin{array}{c}23 / 100 \\
10^{30} \mathrm{~cm}^{-2} \mathrm{~s}^{-1}\end{array}$ & \\
\hline IP beta function $b_{x}$ & $1.75 \mathrm{~m}$ & $1.25 \mathrm{~m}$ & \\
\hline IP beta function $b_{y}$ & $7.0 \mathrm{~cm}$ & $4.0 \mathrm{~cm}$ & \\
\hline
\end{tabular}

advance and tunes values) were also changed in attempts to optimize the emittance and the beam-beam behavior.

With the increase in energy to above the $\mathrm{W}$ pair threshold, the beam-beam limit increased, and the challenge was to develop a low emittance optics with sufficient dynamic aperture to go to the $100 \mathrm{GeV}$ regime. Luminosity production was maximized by increasing the bunch current to the limit while operating with four bunches per beam and rigorous optimization of vertical and horizontal beam sizes.

Between 1996 and 2000 the beam energy was progressively increased from 80.5 to $103 \mathrm{GeV}$. At these energies beam oscillations are strongly damped and the single particle motion has an important random walk component due to the large number of emitted photons. Consequently, particles no longer lock on resonances driven by the nonlinear beam-beam force and beam size blowup is reduced allowing the use of higher bunch currents. Record beam-beam tune shifts of above 0.08 were achieved in each of the four collision points. In order to reach these very high energies, the superconducting cavities were all driven beyond their design values to reach a total accelerating voltage per turn of more than $3.6 \mathrm{GV}$ (see Fig. 25 for the progression in accelerating voltage).

The design and achieved values for a number of crucial LEP performance parameters are summarized in Table III. It is seen that LEP clearly surpassed all design expectations. In particular the peak luminosity at LEP2 was almost a factor of 4 above design.

Competition from the USA. - LEP was not the only game in town. There was fierce competition from the more innovative Stanford Linear Collider (SLC) in California. But LEP got off to a fantastic start and its luminosity increase was much faster than at its relatively untested linear counterpart.

A short article capturing the transatlantic rivalry appeared in the Economist on 19 August 1989.

"The results from California are impressive," the magazine reported, "especially as they come from a new and unique type of machine. They may provide a sure answer to the generation problem before LEP does. This explains the haste with which the finishing touches have been applied to LEP. The $27 \mathrm{~km}$-long device, six years in the making, was transformed from inert hardware to working machine in just four weeks-a prodigious feat, unthinkable anywhere but at CERN. Even so, it was still not as quick as Dr. Carlo Rubbia, CERN's domineering director-general might have liked."

Performance summary.-The performance of LEP was fundamentally limited by the nonlinear forces of the beambeam interaction (the "beam-beam limit"). The beam-beam limit was characterized by the linear part of the force "the beam-beam tune shift." Over many decades, accelerator physicists had been battling with the beam-beam limit shift as it had a one-to-one relationship with the specific luminosity. Before LEP, the highest beam-beam tune shift ever attained was 0.045: in LEP a maximum value of 0.083

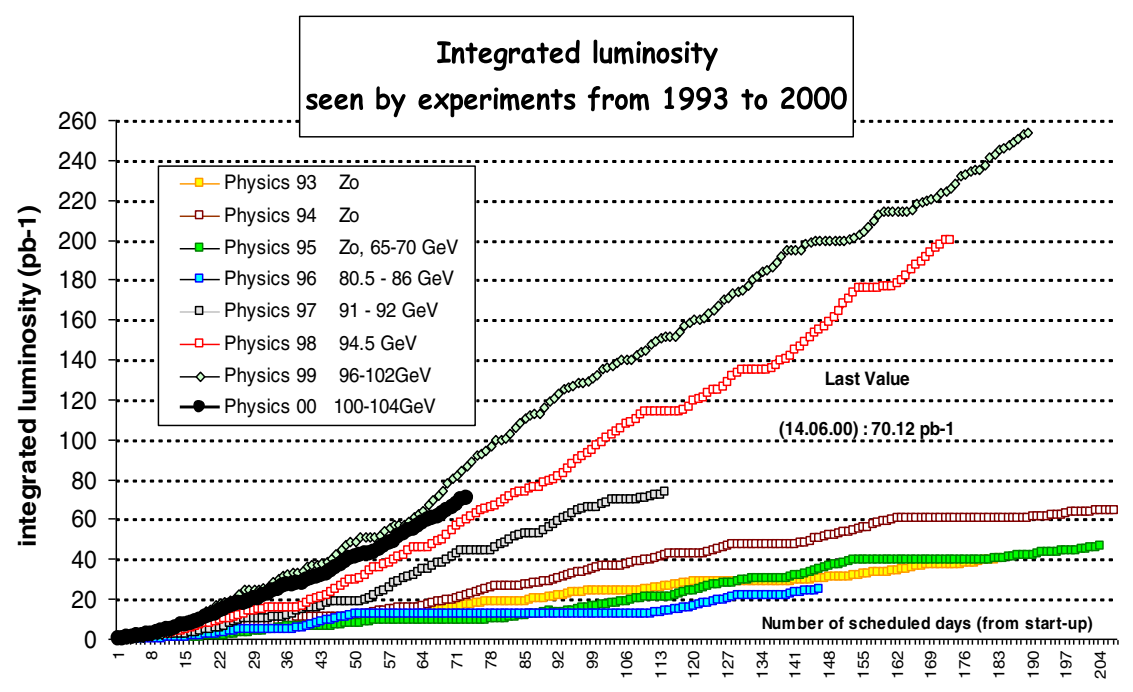

FIG. 26. LEP daily integrated luminosity from 1993 to 2000. 
was reached at high energies almost certainly due to the very fast synchrotron damping time at these high energies.

The luminosity performance of LEP over the eight-year period 1993-2000 [23] is shown in Fig. 26. This performance was even more impressive given that the operational mode was changed every single year.

\section{Insertion: 1991, The first Chamonix performance workshop}

When we first started working on the LEP accelerator (in the late 1980s), I was invited to an experimental particle physics retreat. It was in the mountains (Les Arcs in the French Alps), where we worked in the mornings, skied in the afternoons, worked in the evenings and then had dinner together. It struck me that this was a really excellent way to build teams, and to break down barriers related to cultural or scientific expertise. So, in the autumn of 1990, based on this earlier experience, I proposed a first one-week long retreat in the mountains. We set some requirements for the location and started looking at possible sites both in Switzerland and in France. The best offer we received was from a family run hotel called La Prieurie in the heart of Chamonix and an offer from the "Majestic," a beautiful old Chateau transformed into a seminar center. For this first meeting we were between 65 and 70 attendees.

The workshop dates were set for February, and the format was a direct copy from les Arcs. The discussion topics were split in two halves: analysis of the results and performance from the previous year and agreement on the way to operate the collider in the coming year. The discussions were lively and animated and surprisingly, I noticed people skiing with each other who had hardly even before spoken to each other, even though they worked on the same project, LEP. I even noticed some people who were known sworn enemies actually having a coffee together on the slopes around Chamonix. The workshop was a huge success and all of the main participants returned to CERN knowing exactly how we were going to run and optimize LEP during 1991.

Following the success of the first LEP workshop (we deliberately called it the First Chamonix to imply that there would be many more), "Chamonix" as it has become known became an annual event throughout the LEP years and the LHC years ("Chamonix" is still taking place even after my retirement from CERN).

Short history of LEP2. - In 1995 a major upgrade began (LEP2), enabling LEP to reach beam energies above the WW threshold of $161 \mathrm{GeV}$. I was appointed LEP2 Project Leader by the CERN Council in 1996 with overall responsibility for the construction and operation of the LEP2 project. The major technical part of the project was the construction and commissioning of 288 superconducting cavities. LEP2 was a major success and the design parameters were exceeded in both luminosity and maximum achieved beam collision energies.
The maximum LEP2 energy reached was $104.4 \mathrm{GeV}$ per beam, which required a total rf voltage per turn of more than $3.5 \mathrm{GV}$ to replenish the energy lost due to synchrotron radiation of more than $3.1 \mathrm{GeV}$ per turn. There was also a continuous effort to increase the luminosity by increasing the intensity (number of bunches), reducing the emittance by increasing the overall focusing, and focusing the bunches more tightly at the interaction points. The performance of LEP was ultimately limited by the nonlinear forces of the beam-beam interaction-the perturbation of the beams as they cross the opposing beam.

\section{Insertion: 2000, The last year of LEP2 operation: On the verge of a great discovery?}

LEP's days were never fated to dwindle. Early on, CERN had a plan to install the Large Hadron Collider in the same tunnel, in a bid to scan ever higher energies and be the first to discover the Higgs boson. However, on 14 June 2000, LEP's final year of scheduled running, the ALEPH experiment reported a possible "Higgs" event during operations at a center-of-mass energy of $206.7 \mathrm{GeV}$. It was consistent with "Higgs-strahlung" whereby a $\mathrm{Z}$ radiates a Higgs boson, which was expected to dominate Higgs-boson production in $\mathrm{e}+\mathrm{e}-$ collisions at LEP2 energies. On 31 July and 21 August ALEPH reported second and third events corresponding to a supposed reconstructed Higgs mass in the range $114-115 \mathrm{GeV} / c^{2}$.

LEP was scheduled to stop in mid-September with two weeks of reserve time granted to the LEP experiments to see if new Higgs-like events would appear. After the reserve weeks, ALEPH requested a two months extension to double its integrated luminosity. One month was granted, producing a $50 \%$ increase in the accumulated data, and at the LEPC meeting on 10 October, ALEPH presented an update of their results: the signal excess has increased to $2.6 \sigma$. Things were really heating up, and on 16th October L3 announced a missing energy candidate. By now the accelerator team we were pushing the collision energy of LEP to its limits, to squeeze out every ounce of physics data for the experiments' search for the elusive Higgs. At the LEPC of 3 November, ALEPH presented new data which confirmed their excess once again-it had now grown to $2.9 \sigma$. A request to extend LEP running by one year was made to the LEPC. There was gridlock, and no unanimous recommendation could be made.

Everyone remotely associated with CERN was discussing the proposed running of LEP2 in 2001 to get final evidence of a possible discovery of the Higgs' boson. Arguments against included delays to the start of the LHC of up to 3 years. There was also a worry that Fermilab's Tevatron would beat the LHC to the discovery of the Higgs. There were other more mundane but realistic and practical arguments associated with the transfer of human resources from LEP to the LHC, as well as the impact on the materials budget, including electricity costs etc. The impending 


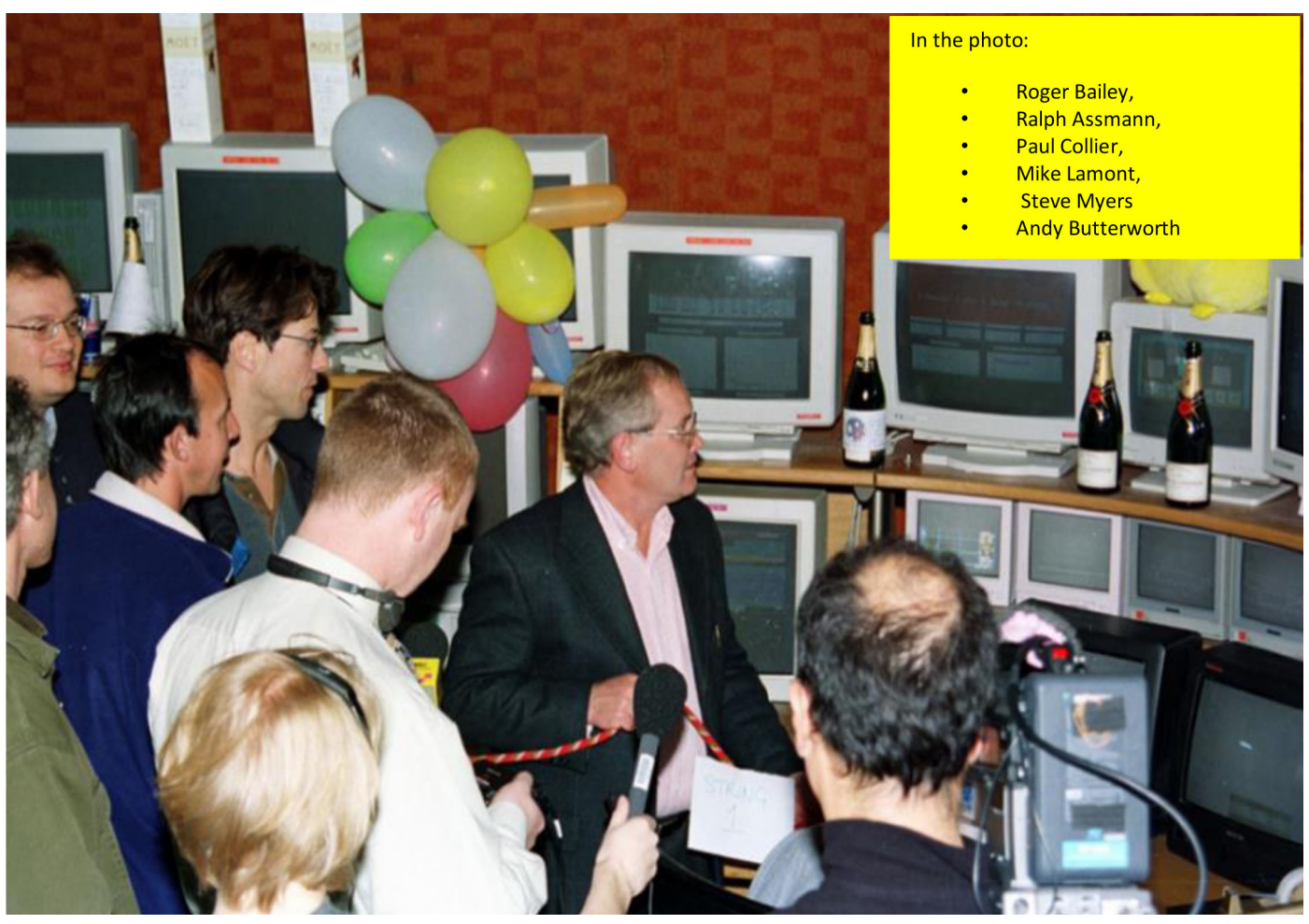

FIG. 27. Last beam dump in LEP.

closure of LEP, when many of us were sure that we were about to discover the Higgs, was perceived like the death of a dear friend by most of the LEP-ers. After each of the public debates on the subject a group of us would meet in some local pub, drink a few beers, curse the disbelievers, and cry on each other's shoulders.

This was the one and only "civil war" which I have ever seen in my 43 years at CERN.

The CERN Research board met again on 7 November and again there was deadlock, with no unanimous recommendation, the vote being split 8-8. The next day CERN Director-General Luciano Maiani announced that LEP had closed for the last time. It was a deeply unpopular decision, but history has shown it to be correct, the Higgs being discovered at the LHC 12 years later, with a mass of not 115 but $125 \mathrm{GeV} / c^{2}$.

When LEP was finally laid to rest most of the LEP protagonists went into deep mourning, and we met one last time for an official wake (see Fig 27, CERN CDS photo 010405).

LEP closure in 2000 and transfer of personnel to the LHC.-The LEP collider was closed in 2000 and decommissioned to prepare for the installation of the LHC collider in the same tunnel.

After the machine was dismantled, requiring the removal to the surface of around 30,000 tonnes of material, some of the magnets and rf units were shipped to other labs for use in new projects. Today, LEP's concrete magnet casings can still be seen scattered around CERN as shielding units for antimatter and fixed-target experiments, and even as road barriers. The quality and precision of the physics data remains unsurpassed in luminosity, energy and energy calibration. It is the reference for any future e $+\mathrm{e}$--ring collider design.

The legacy of LEP. - LEP was the highest energy e $+\mathrm{e}-$ collider ever built. It may well be the last such accelerator ever to be built. The legacy of LEP was and is extremely important for present and future colliders. The quality and precision of the physics data is unsurpassed. (luminosity, energy, energy calibration). LEP is the reference for any future $\mathrm{e}^{+} \mathrm{e}^{-}$ring collider design. The list of contributions to future machine and the legacy of LEP is given (see for example [23]): (i) the physics data on the $\mathrm{Z}$ and $\mathrm{W}$ (luminosity, energy, energy calibration), (ii) the ultraprecise beam energy determination, (iii) operation at record beambeam tune shifts, (iv) the experience in running a very large collider (technical requirements to control a large-scale facility, operational procedures for high efficiency, orbit optimization in long machines, alignment, ground motion and emittance stability in deep tunnels), (v) designing and efficiently operating a large SC rf system, (vi) impedance and transverse mode coupling instability (TMCI) evaluation in large colliders, (vii) flexibility in beam optics designs with changed in the betatron phase advance per cell ranging from $60^{\circ} / 60^{\circ}$ to $102^{\circ} / 90^{\circ}$ and $102^{0} / 45^{0}$, (viii) strong reminder of the need for quality beam instrumentation and controls for efficient commissioning, (ix) the use of the personnel experience and expertise 
gained in LEP/LEP2 to prepare beam commissioning and operation of the LHC collider, and (x) avoid tunneling in nonsolid rock terrain.

The experience gained for the first time in running a very large collider was invaluable for the preparation of the LHC. The importance of shutdown planning, cold checkout, preventative maintenance, remote repairs are all important aspects of any future collider.

The real-time feedback on beam parameters (orbit, tune, instabilities etc.) developed at LEP has proven absolutely essential for the safe and efficient operation of the LHC.

The experience in operating large superconducting and cryogenic systems has also proved invaluable for the LHC operation.

\section{J. Moving focus and personnel to the LHC}

The closure of LEP allowed massive redeployment of skilled and experienced CERN staff from LEP2 to the LHC design.

With the new focus from the closure of LEP, the design of the LHC gathered real momentum with Lyn Evans leading the overall project and the three accelerator department heads leading the technical design of the components: Philippe Lebrun (magnets, cryogenics and vacuum), Paolo Ciriani (infrastructure and technical services), Stephen Myers (radio frequency, accelerator physics, beam diagnostics, controls, injection extraction and beam dump, machine protection, and power supplies). It is important to highlight the enormous contributions of the CERN technical groups and group leaders in this huge effort. The strength of CERN is imbedded in these technical groups.

\section{Insertion: Wolfgang Schnell (1929-2006)}

Wolfgang Schnell was my mentor and an inspiration for me. He was diagnosed with terminal cancer in 2005/2006 at the age of 76/77. I went to visit him on his death bed at his Geneva home, in the summer of 2006, after the closure of LEP and during the installation and testing of the LHC. We had a long chat about the "old days" in the ISR.

Wolfgang died on the 2nd of October in 2006. In his obituary [24] was written:

“...Wolfgang was one of CERN's pioneers and made numerous significant contributions to the field of accelerator physics and technology throughout his career.

...in 1959, he achieved a breakthrough during the running-in of the PS, which suffered from substantial beam loss during acceleration. With the phase-lock feedback system built by Wolfgang, the beam went immediately to $24 \mathrm{GeV}$ with hardly any losses. He was proud to show later that the electronics of his system had been built inside a coffee tin.

...Wolfgang then became a member of the design team that studied the next generation of CERN accelerators after the PS - the Super Proton Synchrotron (SPS) and the
Intersecting Storage Rings (ISR)—and made significant contributions to both.

He proposed a traveling-wave structure for the $300 \mathrm{GeV}$ synchrotron for the acceleration of the particles, which are nearly relativistic at injection energy. Such a structure is still in use in the SPS, faithfully accelerating protons and ions since 1976.

...In 1983 the ISR was closed in favor of LEP, and Wolfgang was one of the leaders of the initial study group. He went on to be the driving force of the LEP rf group, which constructed the largest and most complex rf system in the world based on copper cavities. In the same year, he was the first (with Stephen Myers) to present a paper on the parameters of a future proton-proton collider in the LEP tunnel (the present LHC) and participated actively in the brainstorming on CERN's future in 1985.

...Many will be forever proud to have been in one of his teams and to have had the honor to work with him. He will be sorely missed."

2008 CERN Director of Accelerators and Technology (DAT)

The Director General of CERN has a normal mandate of five years. The mandate of the incumbent Director General, Robert Aymar, was due to terminate at the end of 2008: it had not been a happy coexistence between Aymar and the Council particularly in the last two years of his mandate. Aymar and I did not see eye-to-eye on many topics. He was a very authoritarian engineer who was educated in the elite École Polytechnique, of France.

The CERN Council had already nominated the incoming DG; he was Rolf Heuer, who had previously been the spokesperson of the Opal collaboration in LEP, and we had worked together for many years during LEP operation.

Sometime in early summer 2008, Rolf arranged a meeting with me, and explained that he was in the process of setting up his new directorate for approval by CERN Council in the autumn. He explained that he wanted to have a team who would work together in harmony and that he already had positive experience with me while I oversaw LEP. He then asked if I would be interested in being nominated as the Director of Accelerators. I was both surprised and of course flattered. Surprised because by the beginning of his mandate in 2009, I would be 62 years and 5 months old, and CERN has a compulsory retirement age of 65 years for all staff. I explained this to Rolf who replied that he was aware of this but that I could do half of his mandate and retire in August 2011. I agreed to be nominated, and he then asked me to think about the structure of the accelerator sector and possible names for nomination of Department Heads.

LHC start-up 10th September 2008-Exhilaraton.-As department head of the CERN Accelerator and Beams department, it was my responsibility to direct the commissioning and operation of the LHC collider. 
The intended start-up of the LHC had received enormous media attention. Television and radio companies from all over the world were contacting CERN for an official startup date. On the day of the first beam, it was almost impossible to get near a console to try to operate the collider due to the crowds of CERN interested staff and the media mob.

Nevertheless, it was a magnificent day for CERN and all the years of work that had gone into making this fantastic instrument.

On that day, CERN put basic science firmly on the public agenda. The statistics were impressive: 300 journalists on site, over $2000 \mathrm{TV}$ broadcasts, 100 million hits on the CERN website, and an estimated global audience rivalling the number of particles in the LHC's first beam.

At the end of the day, one journalist asked whether we had taken a risk in inviting the world to witness such a delicate operation. It certainly had been a risk, but a risk that paid off handsomely.

Below is a short summary of the events of 10th September 2008.

Although everybody was hoping to see the first circulating beam at some point in the morning, very few expected that it would happen so smoothly. The aperture was clear, the magnets all had the correct polarity, and the beam went from one sector to the next and a complete turn was made in less than an hour. Note the actual time for the beam to cover the $27 \mathrm{~km}$ is less than one ten-thousandth of a second.

At 10:28, when the first beam was steered around the machine, the applause resonated loudly throughout the main auditorium. The explosion of joy and pride erupted simultaneously as all the conference rooms were connected via webcast to the CERN Control Centre (CCC).

Around 15:00 another beam of protons circulated in the second-anticlockwise-ring. Again, this happened very smoothly. There was a little hiccup at the end of the morning, which for me was a gentle reminder of the opposite sort of day it could have been.

The accident 19th September 2008: Despair.Following the impressive start-up on 10th September, a technical problem arose with an electrical transformer which necessitated stopping commissioning for a few days. During this stop it was decided to test the last octant (sector 34) up to $9.3 \mathrm{kA}$, i.e. $10 \%$ above the dipole current required for operation at $5 \mathrm{TeV}$ per beam. At $8.7 \mathrm{kA}$ a resistive zone developed in the dipole bus-bar magnet interconnects. This led to thermal runaway in one of the magnet interconnects, followed by the development of an electrical arc, initially across the interconnect, later puncturing the helium enclosure, and finally puncturing both beam pipes. The release of the helium caused a pressure wave over a region of more than 400 meters resulting in damage to magnets, interconnects and pollution of the ultrahigh vacuum system.
We did the first turn with the LHC on 10th September 2008, when the world's press was in the control room and the mood was one of euphoria. Nine days later, the accident happened, and it seemed inevitable to me that we would have unbearable scrutiny from the journalists who had covered the story. From a technical point of view this could be compared to the space shuttle disasters with the important difference that no-one was killed or injured.

As it happened, however, another even more important disaster took place only days before the LHC accident: the bankruptcy of Lehman Brothers (announced 15th September, just three-four days before the LHC accident!).

This was the largest bankruptcy filing in the history of the U.S. with Lehman holding over $\$ 600$ billion in assets, and of course took most of the journalists' attention. From a media point of view, the LHC accident went by fairly unnoticed.

One of my current colleagues (an ex-banker) who worked at Lehman at the time, often quips "You see Steve, the lengths I went to, to help a friend."

Following the accident, Aymar and Evans published a statement in the CERN Bulletin of 6 October, long before a proper analysis had been made, saying that

"The LHC will be started up again in spring 2009 following the winter shutdown for the maintenance of all the CERN installations ...."

Evans was also quoted in the same issue "We are not worried about repairing the magnets as spare parts are available."

Before the accident, an official ceremony for the inauguration of the LHC with Heads of State and Ministers from CERN's member states was organized to take place on 21st October and followed by an LHCfest. Aymar and Evans decided to continue with this celebration despite the fact that the LHC was severely damaged and inoperable.

Post-mortem of the accident.-An inquiry by CERN specialists [25] indicated several causes of the substantial damage to the machine:

There was an absence of solder on the offending magnet interconnect giving a contact resistance of $220 \mathrm{n} \Omega$ (design $\sim 1 \mathrm{n} \Omega$ ).

There was poor electrical contact between the superconducting (SC) cable and the copper stabilizing busbar.

The fault detection of the interconnect was not sensitive enough. If the fault detection had been more sensitive, the accident would have been prevented.

The pressure relief ports were under-dimensioned for an accident of this magnitude.

The anchorage of the magnets to the tunnel floor was inadequate.

In the LHC, the maximum current flowing in the coils of the superconducting magnets is $13.5 \mathrm{kA}$. This produces a huge amount of stored energy: 11 gigajoules, about the same as a Nimitz-class aircraft carrier going at full battle speed. If something goes wrong, that energy must be 
automatically transferred for safe dissipation outside the magnets. The design is to extract this energy and dissipate it in the resistors that form the magnet protection system. For a magnet to be superconducting, it has to be below a certain temperature, typically around 4 Kelvin (minus 267 degrees centigrade). The magnets are 15 meters long, and there are 1,232 of them. When the coil is superconducting, it has almost zero resistance, so the current flows without resistive heating. If the temperature rises above the critical temperature then the coil becomes nonsuperconducting, and becomes more resistive. This resistance in the presence of 13,500 amps causes heating, which in turn causes the resistance to grow: a vicious destructive spiral, known as a "quench." The machine protection is designed to protect the magnets in case of a quench.

One of the sources of heat can be the movement of the coils, which can create friction. Another potential source of heat is the proton beam itself, which can deposit a huge amount of energy

The LHC accident occurred because the magnet protection system did not work. There was a resistance runaway, and the energy was not transferred out of the magnets fast enough. The huge energy remaining in the magnets caused a huge electrical arc (like a continuous bolt of lightning, or an electric arc welder), which melted more and more of the copper. As the electric arc lengthened, then the distance to another electrically earth component became less than the arclength; like a lightning bolt which always finds the closest distance to earth. Quickly, the electric arc now reached the cryogenic line. The helium inside the cryogenic line suddenly went from minus 269 degrees to room temperature in an instant, expanding dramatically. The pressure release valves were under-dimensioned, and could not release the pressure rapidly enough, so the pressure built up inside the magnets. All the magnets in the area were ripped off their anchor points, ripping out the concrete anchors that were holding them in place.

Some of the worst damage, however, was to the vacuum, which was suddenly increased to atmospheric pressure, and therefore sucked in all the debris which had been created in the explosion. The whole process probably lasted a very short time.

The damage inside the tunnel was impressive but horrific: many large heavy components had been blasted out of their places by the force of the "explosion." We were very concerned about inspecting the magnets due to their weight and their precarious positioning after the accident. For the repair, replacing the magnets was of course much more complicated than installing them for the first time, because they had to be extracted from the full tunnel, in the restricted space with little room to maneuver.

Organizing the repair.-As newly nominated Director of Accelerators and Technology, it was clearly my responsibility to direct the repair of the LHC. I decided to organize the repair through the structure of the LHC Machine
Committee and the Chamonix annual retreats, since all the required specialists were already involved in these meetings.

Chamonix 2009: The repair.-Following the initial investigation of the resulting damage, a crash program was set up to repair and consolidate the LHC. The teams included many CERN partners, collaborators, detector people as well as the accelerator sector.

I also created two new external panels, the first on Technical Risk was headed by Don Hartill from Cornell, and the second on Quench Protection headed by Jay Theilacker from Fermi National Accelerator Laboratory. Most of the members of these panels participated in the Chamonix meeting. We also initiated a safety group plus a task force to analyze the accident.

The task was enormous and required not only the repair of the damaged components but equally importantly reengineering many elements so that a similar accident would never occur in the future. A total of 39 dipole magnets were replaced. In addition, 14 quadrupole magnets were replaced and a total of 54 damaged magnet interconnects needed full repair with around 150 extra needing partial repair. About $5 \mathrm{~km}$ of ultrahigh vacuum beam tube required the removal of small pieces of superinsulation and black soot followed by careful cleaning. A new longitudinal magnet restraining system was designed and installed on 50 quadrupole magnets. All existing flanges on the magnets were equipped with additional pressure relief ports (typically $10 \mathrm{~cm}$ diameter) and $20 \mathrm{~cm}$ flanges were cut on dipoles and equipped with double size pressure relief ports. In total 900 helium pressure relief ports were added. A major task was the upgrade of the magnet protection system which had been shown to be insensitive as a protection of the interconnect splices. The new design is 3000 times more sensitive than the older system and involved 6500 new detectors and the installation of more than $250 \mathrm{~km}$ of cable in the LHC tunnel. A major added advantage of the new magnet protection system is that it provides continuous measurement, to sub-n $\Omega$ precision, of the resistance of all intermagnet splices in the machine. This allows continuous monitoring of the quality of the interconnects and early warnings of possible problems.

Among the other issues studied at Chamonix was the road map and schedule for the LHC operation over the next years. The planning was a nightmare due to the strong interdependences of many of the critical systems: the cooling and ventilation and the cryogenic maintenance, the inadequate helium storage capacity, the unidentified defectuous plug-in modules (which compensated for the contraction and expansion of the LHC circumference during cooldown and heat up) and the electrical quality assurance. In addition to these technical problems it had been decided in the past that LHC would not run during the winter period due to the increased cost of electricity. A change in this strategy would incur an increased electrical 
cost of 8MEuros and impacted heavily on the scheduled shutdown work planned in CERNs other accelerators which were needed as injectors. There was also a big impact on standard annual maintenance of the water-cooling towers and the electrical network. The CERN committees adopted the first proposal which was to plan electricity provision for the 2009-2010 winter and exceptionally operate the collider through the winter months.

\section{K. Chamonix January 2010}

After the Christmas break it was time for the Chamonix retreat once again. This time we had, amongst others, two very hot topics; the beam energy scenario for the LHC and a project proposal to replace the injector chain of the LHC by a new superconducting linac and an upgraded proton synchrotron.

For the LHC, two energy scenarios were compared. The first was to run at $3.5 \mathrm{TeV} /$ beam to accumulate as much data as possible at this energy, and to delay the consolidation of the whole machine for $7 \mathrm{TeV} /$ beam in the foreseen long shutdown 1 (LS1).

This first option would mean that we would be entering the longest phase of accelerator operation in CERN's history, scheduled to take us into summer or autumn 2011. For the experiments, these 18 to 24 months will bring enough data across all the potential discovery areas to firmly establish the LHC as the world's foremost facility for high energy particle physics.

What was the reasoning behind this proposed change in conventional operational mode of the CERN accelerators? Firstly, the LHC is unlike any previous CERN machine. Because it is a cryogenic facility, each run is accompanied by lengthy cooldown and warm-up phases. For that reason, CERN's traditional "run through summer and shutdown for winter" operational model had already been brought into question. Furthermore, we have known for some time that work is needed to prepare the LHC for running at energies significantly higher than the $7 \mathrm{TeV}$ collision energy we have chosen for the first physics run.

The second option was to run until the second half of 2010 then do the minimum repair on splices to allow $5 \mathrm{TeV} /$ beam in 2011 ( $7 \mathrm{TeV} /$ beam comes much later).

The discussion on the choice of these two options was heated and emotional. Some people openly disagreed with either of the two options and insisted that LHC had been tested and proven up to $5 \mathrm{TeV}$ per beam and operation in 2010 should be at this energy. They dismissed and ignored the measurements and simulations which clearly showed that operating at $5 \mathrm{TeV}$ was highly risky. Like most of my colleagues, I was totally convinced that this $5 \mathrm{TeV}$ proposal was not only risky but foolhardy. I was also convinced that if we ever had a second accident like that of 2008, CERN's future would be jeopardized. Fortunately, common sense prevailed, and the experiments agreed to follow the first of the official proposals which was the lower risk for data taking.

\section{Insertion}

As a result of measurements done, some years later, during LS1, it was clearly shown that if we had decided to run LHC at $5 \mathrm{TeV}$ in 2010 we would almost certainly have provoked another serious accident.

The second major topic was the replacement of the injector system for LHC. This was the second phase of a larger project under Aymar which had also included replacement of the existing LINAC2 by a new LINAC4. I was not in agreement with these projects as I could not justify them from the LHC performance point of view. In my opinion these new projects were not being proposed for the LHC performance but for other unmentioned proposals like neutrinos. I could not halt LINAC4 since the construction was well under way, but I had asked the injector specialists for a clear objective comparison of the performance limits for the LHC, between an upgrade of the injector systems and their replacement. The result was very clear: the proposed replacement was highly more resource intensive, much riskier and would not give any clear performance improvement in the LHC when compared with the much simpler less risky upgrade of the existing and well tested present injectors. The LHC Injector Upgrade (LIU) was born as a result. As always, Chamonix had been a tough but very useful and productive retreat.

First LHC $7 \mathrm{TeV}$ collisions after the repair 2010 (from CERN bulletin 06-07/2010 Monday 8 February 2010).The first collisions at $7 \mathrm{TeV}$ center-of-mass energy were recorded on March 30, 2010. During 2010, operation was continuously divided between machine studies to increase the luminosity and physics data taking.

We had already suffered from the stored energy in the magnet system which produced the accident in 2008, however many of us were more concerned about the stored energy in the beams. Although the amount of stored energy in the beams was much lower than that in the magnets, the type of energy in the beams was potentially much more destructive. A single beam of full intensity at high energy had enough energy to evaporate $500 \mathrm{~kg}$ of copper. In addition, the cross section of the beams at the collision points was smaller than a human hair. Consequently, any uncontrolled beam loss had all the potential for creating a very long thin hole in the LHC. Beam losses could be provoked by any of the thousands of components in the LHC as well as events outside our control like lightning strikes on the electrical supply lines. Add to this the fact that the beam was surrounded by $22 \mathrm{~km}$ of superconducting magnets at a temperature of $-271 \mathrm{C}$. No one really knew for sure what the destructive power of a $7 \mathrm{TeV}$ beam would be, simply because no one had ever operated such a beam. Many computer simulations had been done for the design 
of the beam dump (which is where the beam is targeted in a beam abort), and we had asked for some input from the U.S. military, but the answer was always the same: we do not have any experience.

The LHC beam protection system is the most intricate accelerator protection system ever developed. It has literally thousands of beam abort triggers and relies on very stringent control of the optics of the machine, both locally and globally. The collimation system is part of the machine protection system and must intercept almost the totality of any beam losses if they are to protect the rest of the machine. When the collimators are well set up and the hierarchy of losses is correct, the vast majority of all losses are localized at the collimator.

The rate of progress was impressive, nevertheless before each step was taken to increase the intensity and hence the stored beam energy, all machine protection systems were validated up to the new higher level.

In 2010 we proceeded with great care and when the CMS experiment reached the tiny (by today's standards) integrated luminosity level of $1 \mathrm{pb}^{-1}$, they showed that their experiment had already reproduced all the physics discoveries of the previous century. This was a clear demonstration of the discovery power of the LHC. In the second half of 2010, the performance rate of the collider increaser enormously and a final integrated luminosity of $44 \mathrm{pb}-1$ was recorded by the experiments.

2011 prolongation as DAT “Lex Myers".-In Heuer's initial proposal to the CERN Council, my mandate was to end on my 65th birthday which was on the third of August in 2011. In the CERN employment conditions, no staff member could continue as a staff member after the age of 65 .

I do not know who took the initiative to propose my extension, whether it was Rolf Heuer or the CERN Council. In any case, in March 2011, the DG wrote a very comprehensive paper (Confidential: Restricted Distribution; "White Paper: Senior Staff Appointment") justifying my prolongation as DAT and proposing an amendment to the Staff Rules and Regulations.

This change in the staff rules and regulations was nicknamed "Lex Myers" by the CERN lawyers.

The introduction read:

"The current LHC operation as well as its programme for the immediate future require managerial stability and continuity in key positions. The attached draft document contains a proposal to this effect, which is submitted for discussion and endorsement in principle by the Council in preparation for the formal submission of the proposal for approval in June 2011."

In this paper, the Council was invited:

"to approve the proposed amendment to the Staff Rules ... and to approve the extension of the term of office of Dr Stephen Myers as Director for Accelerators and Technology and his employment contract until the end of the mandate of the current Management on 31 December 2013."

The justification for the setting of this precedent was highlighted in the document to Council:

".... CERN is at major cross-roads in its history. ... in the light of the 2011 Chamonix LHC Workshop, to propose ... an extension of LHC operation at or around $3.5 \mathrm{TeV}$ per beam until the end of 2012, the discovery potential of the LHC and its reliable operation over the coming 18 months will be critical not just for CERN and its reputation but also for particle physics as a whole.

...At the present critical juncture for the Organization, the Management considers that continuity is essential within the AT Sector management team and Dr. Myers' replacement would not be in the Organization's interest at this pivotal moment. ...

...With the support of the Accelerator Sector management team, the Director-General is therefore proposing that the appointment of Dr Myers as Director for Accelerators and Technology be extended until the end of the mandate of the current Management in December 2013....
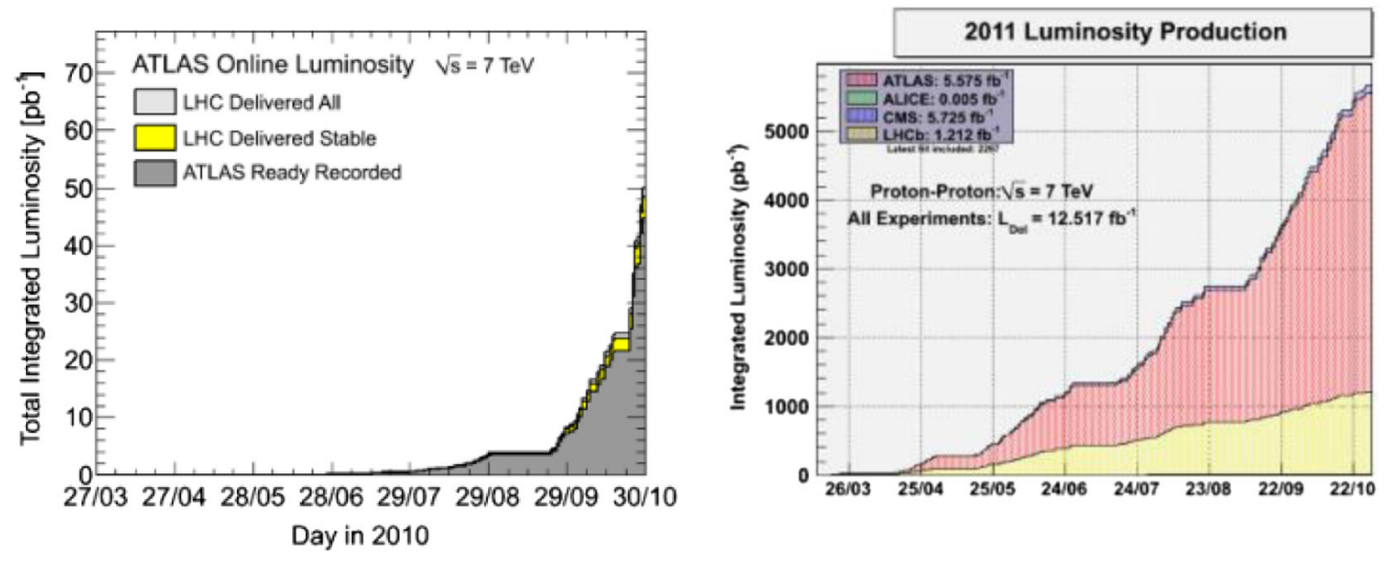

FIG. 28. Plots show the daily progress in performance of the LHC in 2010 and 2011, reaching a maximum of 44 in 2010 and 6000 in 2011. 
Council accepted the proposal for the extension and changed the CERN employment rules accordingly.

\section{2011-2012 LHC operation [26-28]}

We continued to operate the collider in 2011 and were now increasing the performance much more rapidly (see Fig. 28 comparing 2010 performance with 2011: note the different vertical scaling).

During the second half of 2011, the physics world was waiting for 2012; the last year of LHC operation before the long shutdown. The looming question was: will the LHC discover the Higgs boson or prove that it does not exist? By this time I had enough confidence in my performance simulation program to make predictions of the collider performance in 2012. I ran the program retroactively for 2011 and the results were impressive: it predicted fairly accurately the actual performance achieved in 2011. However this was not totally unexpected as some of the input for the predictions came from 2010 and 2011. Using the daily operations schedule for 2012 and the agreed increase in beam energy to $4 \mathrm{TeV}$ per beam, I ran the simulation and the results were intriguing. The prediction was for a total performance figure of around $16000\left(\mathrm{pb}^{-1}\right)$ and reaching more than 7000 before the June technicals stop. The best estimates for a "five sigma" discovery of the Higgs was around 13000. According to the simulation we would either discover or prove the nonexistence of the Higgs before the end of 2012.

The end of the year collaboration meetings were coming up in the CMS and ATLAS experiments and I was on the schedule to make presentations on 21st and 22nd November 2011. I decided that I would "take the plunge" and publicize the results at both these meetings. I showed the estimated performance evolution on a day-by-day basis for all of the scheduled days in 2012. Operation was due to stop in mid-October at which time the simulation predicted an integrated performance of more than 16000, significantly more than needed for the best estimate of discovery.

Operation of the LHC restarted in April 2012 and I was carefully watching the performance every day. Initially the preformance was lagging the prediction, but then during May the slope changed and by the start of the second technical stop on the 17th of June, the actual performance was exactly as predicted (see Fig. 29).

I was now very confident we would reach the threshold for Higgs' discovery before the end of the 2012 run. It normally takes some weeks or months before the experiments can analyze all of the events they have recorded, so I was more than surprised to hear the rumors in late June that the data had been analyzed and there were signs of a Higgs' discovery. The annual high energy physics conference was scheduled for the 4th of July in Melbourne and a video link had been set up from the main CERN auditorium to allow the LHC results to be transmitted to the physics world.

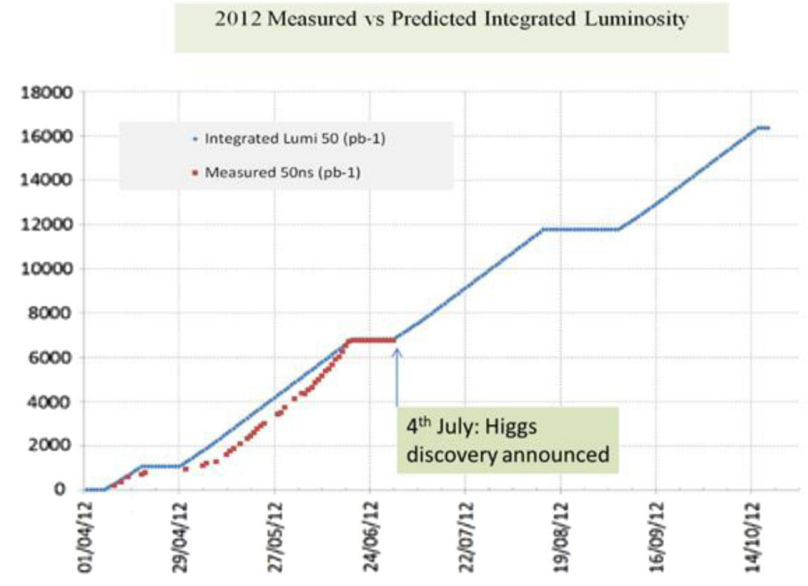

FIG. 29. Plot of predicted integrated luminosity in blue and measured in red.

Everyone in the high energy physics' world was waiting with bated breath. The main auditorium had to be locked to stop the summer students camping out for days beforehand in the conference room and blocking it for anyone else. I arrived at CERN at $5 \mathrm{AM}$ on the morning of the 4th of July and the queue for a place in the main auditorium was already long. Fortunately I had a reserved seat so I did not need to join the queue.

The main auditorium was packed to capacity long before the start of the presentations, with many legendary physicists including Peter Higgs as well as most of the living previous CERN DGs.

CMS had won, by the toss of a coin, to be the first to present. Joe Incandela nervously started his presentation and then, after some explanations on how they analyzed the data, showed the small bump which was the five sigma signal for the discovery of the Higgs. Everyone present stood up and applauded for minutes. Then Joe came over to me sitting in the front row and shook my hand and thanked the accelerator team. He then went back to the microphone and told the audience that he had just thanked us for the great job. I was overwhelmed with pride and joy. After Joe, Fabiola Gianotti made the presentation on behalf of the ATLAS experiment: again a five sigma signal and the auditorium erupted.

Why five sigma? Nearly all physics discoveries are statistical. The "gold standard" for a physics discovery is defined as being at the $5 \sigma$ level of a Gaussian (normal) distribution. In plain terms this means that there is less than 1 chance in 3.5 million of an error. With two independent experiments each having a $5 \sigma$ signal makes the probability much less.

CERN was now triumphant having produced perhaps the most important physics result of the past century. I was also very gratified; I had successfully directed the repair and the operation of the LHC towards its ultimate goal: the discovery of the Higgs' boson. 

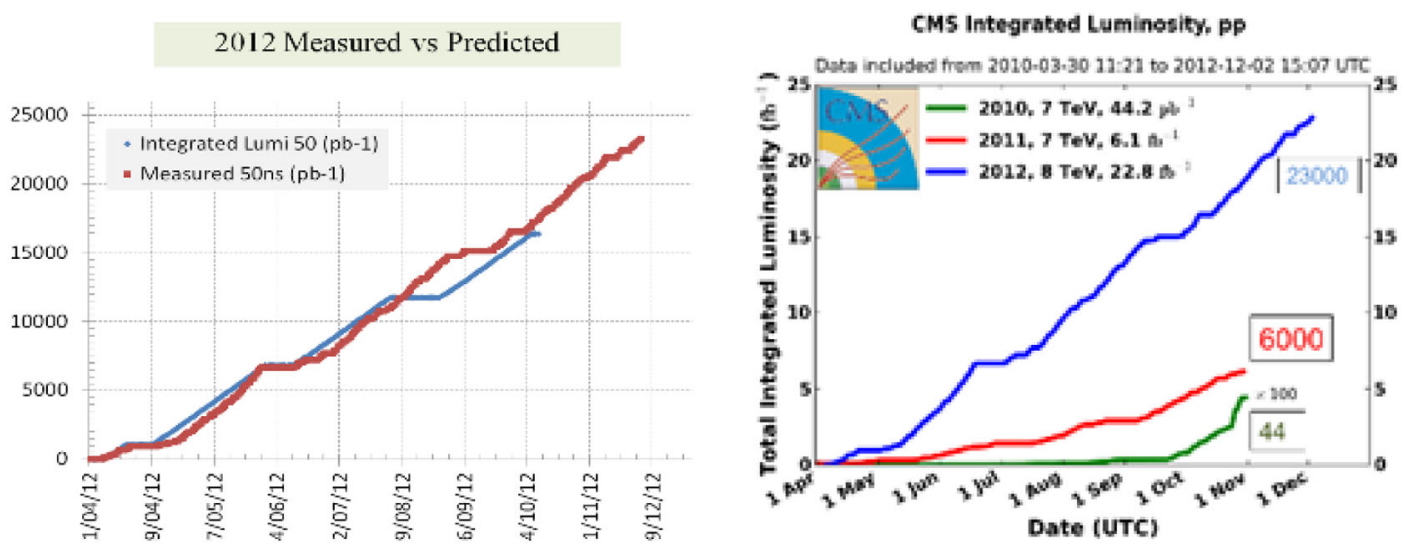

FIG. 30. LHC performance in 2012 (predicted and achieved) and over 2010 to 2012.

After the euphoria of the Higgs' discovery the collider resumed operation with a new approval for a run extension of nearly two months at the end of the year. The final integrated performance at the previously planned end of run was almost exactly at the level of the predictions (see left plot in Fig. 30).

During the three years of operating LHC (see right plot in Fig. 30), the performance increased (in inverse pico-barns, $\mathrm{pb}^{-1}$ ) from 44 in 2010, to 6000 in 2011 and then to 23000 in 2012. An incredible collider!

\section{Insertion: Higgs and LEP2: What might have been!}

LEP started operation in 1989 and continued during 1990. In September 1990 the CERN LEP experiments committee organized a meeting in Cogne, Italy, to review the future of the LEP collider (see program in Table IV below).

I was given the first slot to talk immediately preceded by the opening remarks by the CERN DG Carlo Rubbia.

I was given the task of presenting the future upgrades of the collider. I had prepared slides showing the possible beam energy as a function of the number of installed superconducting $(\mathrm{sc})$ cavities. The maximum number of cavities, in my presentation, went up to a maximum of 384 sc cavities which completely filled all possible spaces around LEP and included the removal of the normal conducting cavities to make space for the more performing sc cavities.

As I gradually moved upwards with the number of cavities, the DG was visibly becoming more and more agitated and kept interrupting my presentation. When I reached the number of sc cavities of 288, the DG told me to stop, as there was no mandate for this presentation. I was completely confused as I had clear instructions from the LEP Experimenters Committee (LEPC) as to what I should present. But I stopped as ordered. My presentation was followed by presentations from the four experiments. The first three suffered the same treatment as I had done, with the DG continuously interrupting and loudly disagreeing.
The only presentation which went uninterrupted was that of L3. Clearly even the CERN DG does not interrupt a Nobel prize winner like Sam Ting!

Following the Monday talks, the DG informed us that he wanted all accelerator people in his office on Friday morning. The people summoned were Carlo Wyss (LEP upgrade project leader), Lyn Evans; SPS-LEP (SL) Division leader, Gunther Plass (Director of Accelerators) and myself (responsible for LEP, and deputy to SL Division leader).

On the Friday morning, the DG was in a state of fury and frenzy. He started off by telling me that what I had done in Cogne was "like putting a bottle of whisky under the noses of alcoholics"! Then he continued screaming and shouting at all the accelerator people, particularly me. I quietly sat and stared into Carlo's face, but I was getting angrier by the second at this unjust admonishment. He ended his attack by declaring that if he had problems at the following Monday's Council meeting, he would demand all of our resignations on his desk by Monday evening. Then he ran out of the meeting room across the corridor to his office.

I immediately jumped up and followed him into his office. I quietly told him that he did not have to wait until Monday evening and that he could have my resignation there and then. He immediately relaxed, put his arm around my shoulder and said, "you are doing a great job Steve, keep it up!!"

Coincidentally that same office was where I spent my five years later as Director of Accelerators and Technology.

For obvious reasons, it was another five years before the subject of filling LEP with sc cavities was ever openly discussed. In 1995 a workshop on physics at LEP2 was organized by Altarelli, Sjostran and Zwirner. Once again, we presented the option for filling all possible locations in LEP with sc cavities and wrote [27]

"By removing all the $\mathrm{Cu}$ cavities and making the layout of Points 2 and 6 identical to that of Points 4 and 8, a total of $384 \mathrm{sc}$ cavities could be installed in LEP, provided that the 16 separators for the Bunch Trains Scheme are removed 
TABLE IV. Discussion program for future of the LEP collider.

\begin{tabular}{|c|c|c|c|c|c|c|}
\hline & & & \multicolumn{2}{|c|}{ COGNE VI $\quad 24-29$ September 1990} & \multicolumn{2}{|c|}{$\begin{array}{l}\mathrm{EF} / 8002 \mathrm{H} / \mathrm{RB} / \mathrm{mm} \\
16 \text { October } 1990\end{array}$} \\
\hline & $\begin{array}{c}\text { MONDAY } \\
24.09 .90\end{array}$ & $\begin{array}{c}\text { TUESDAY } \\
25.09 .90\end{array}$ & $\begin{array}{c}\text { WEDNESDAY } \\
26.09 .90\end{array}$ & $\begin{array}{c}\text { THURSDAY } \\
27.09 .90\end{array}$ & $\begin{array}{l}\text { FRIDAY } \\
28.09 .90\end{array}$ & $\begin{array}{c}\text { SATURDAY } \\
29.09 .90\end{array}$ \\
\hline 09.00 & $\begin{array}{c}\text { Opening remarks } \\
\text { C. Rubbia } \\
\text { LEP } \\
\begin{array}{c}\text { Energy upgrade } \\
\text { (S. Myers) }\end{array} \\
\text { ALEPH } \\
\text { (J. Lefrançois) } \\
\text { DELPHI } \\
\text { (U. Amaldi) }\end{array}$ & $\begin{array}{l}\text { Theory } \\
\text { Comparison of } \\
\text { accuracies } \\
\text { of various LEP } \\
\text { observables }\end{array}$ & $\begin{array}{l}\text { Polarized beams: } \\
\text { 1) Machine } \\
\text { R \& D } \\
\text { 2) Implication } \\
\text { on detectors } \\
\\
\text { LEP/LHC } \\
\text { compatibility }\end{array}$ & $\begin{array}{l}\text { Precision tests and } \\
\text { new physics } \\
\text { Other experiments } \\
\text { LEP 5, Modal } \\
\text { Letters of interest }\end{array}$ & $\begin{array}{l}\text { Heavy flavours } \\
\text { HERA }\end{array}$ & Conclusions \\
\hline 14.00 & $\begin{array}{c}\text { OPAL } \\
\text { (A. Michelini) } \\
\text { L3 } \\
\text { (S.C.C. Ting) } \\
\text { LEP energy } \\
\text { upgrade } \\
\text { (C. Wyss) } \\
\text { Evidence for LEP } \\
\text { polarization } \\
\text { (M. Placidi) }\end{array}$ & $\begin{array}{c}\text { Search for } \\
\text { new particles }\end{array}$ & $\begin{array}{c}\text { LEP performance } \\
\text { on the } \mathrm{Z}^{0} \\
\text { resonance } \\
\text { (S. Myers) } \\
\text { More than } 4 \\
\text { bunches } \\
\text { (J. Jowett) } \\
\text { Implication of } \\
\text { increased number } \\
\text { of bunches on } \\
\text { detectors }\end{array}$ & FREE & $\begin{array}{l}\text { Rare } Z^{0} \text { decays } \\
\text { compositeness }\end{array}$ & \\
\hline 20.30 & $\begin{array}{l}\text { Data analysis at } \\
\text { high luminosity }\end{array}$ & $\begin{array}{c}\text { SLAC } \\
\text { programme } \\
\text { (R.E. Taylor) }\end{array}$ & $\begin{array}{c}\text { B Physics with } \\
\text { proton } \\
\text { machines } \\
\text { (P. Tipton) } \\
\end{array}$ & FREE & (Reserve) & \\
\hline
\end{tabular}

from LEP as well (each separator occupies the location of a four-cavity module), precluding operation with more than four bunches per beam."

Following the 1995 workshop, the decision was taken in 1996 to discontinue the industrial production of the sc cavities.

With 384 cavities LEP could have reached around $223 \mathrm{GeV}$ in the center of mass and possibly discovered the Higgs' boson ten years earlier than the LHC. What might have been!

\section{LHC energy upgrade (FCC)}

As early as 2010, we had started discussing an energy upgrade for the LHC, even though we were still operating the collider at half of its present design energy. This may sound crazy, but the time scale of such projects is decades and the sooner you start the sooner you realize the amount of technological development that is needed.
Frank Zimmermann was the initiator of the first HELHC mini-workshop to be help in October 2010 in Malta which was funded under the auspices of the European Commission. Malta was chosen mainly due to the fact that the Science Advisor to the Prime Minister of Malta was Nicolas Sammut who had previously been a fellow in the LHC division of CERN.

Malta is a beautiful place, but the mini-workshop did not produce a clear direction for the future of the LHC. The proposal discussed was an energy doubling of LHC by replacing the magnets by higher-field magnets. We asked a very prominent theoretical physicist to give us a talk which was supposed to motivate and excite us to continue with this hugely expensive and technically challenging project. Basically, the prominent physicist said, "Doubling the LHC energy would be nice!" For me this was a clear "no go" message. How could we justify spending billions of Swiss Francs on a project that would be "nice" for physics? Frank saw my reaction and totally agreed. We then started 
realizing that a real energy upgrade would need not only higher magnetic fields but also a larger tunnel. A much larger tunnel in the Geneva vicinity seemed out of the question since, in the early eighties, we had maximized the footprint of the LEP tunnel, and it barely fit between the Geneva airport and the Jura mountains.

During the next years we came to the conclusion that the only option for a substantially higher energy supercollider was to find a location for a larger tunnel somewhere in the Geneva area. Learning from the errors of the SSC we were adamant that we should use the existing CERN infrastructure as preinjectors for a new supercollider.

I asked CERN civil engineer John Osborne to try to find a tunnel trace which did not traverse difficult mountain areas, and which had a circumference of at least $80 \mathrm{~km}$, not an easy task in the Geneva area. John came up with three possible sites and engaged specialist companies to identify "geological, hydrogeological, environmental and construction risks for three tunnel layout propositions to establish which layout would be most feasible." The conclusion was that a $47 \mathrm{~km}$ tunnel (lakeside tunnel) bore the minimum risk. However, all three possible had significant tunneling risks.

The European Strategy Group (ESG) was set up in 2006 to define the future of high energy physics over a 5-7-year period. The second meeting was to take place in 2013 , scheduled to coincide with the successful operation of the LHC (and the hoped-for discovery of the Higgs' boson). As Director of Accelerator and Technology, it was my responsibility to prepare the accelerator input for this meeting. The Open Symposium was held in Krakoff with 13 proposals coming from the Accelerator Sector. These proposals included all CERN projects and upgrades of operating facilities. The most far reaching was a draft proposal for a High Energy LHC. In preparation for the Krakoff meeting, a report had been written [29] by seven CERN staff members with the HE-LHC proposal. In this report it was written

"In case of the $80 \mathrm{~km}$ ring, a new optimization space to explore is open. At this stage we can only envisage the following possibilities of collision energy as a function of the dipole field: (1) $42 \mathrm{TeV}$ center of mass (c.m.) with $8.3 \mathrm{~T}$ (present LHC dipoles), (2) $80 \mathrm{TeV}$ c.m. with $16 \mathrm{~T}$ (high field based on Nb3Sn), (3) $100 \mathrm{TeV}$ c.m. with $20 \mathrm{~T}$ (very high field based on High Temperature Superconductors).

This third mentioned possibility has since become the "Future Circular Collider" (FCC), which is now the major project for the long-term future of CERN.

Table $\mathrm{V}$ indicates the chronological evolution of the world's high energy colliders from 1971 to the present time.

TABLE V. Chronological evolution of the world's high energy colliders from 1971 to the present time.

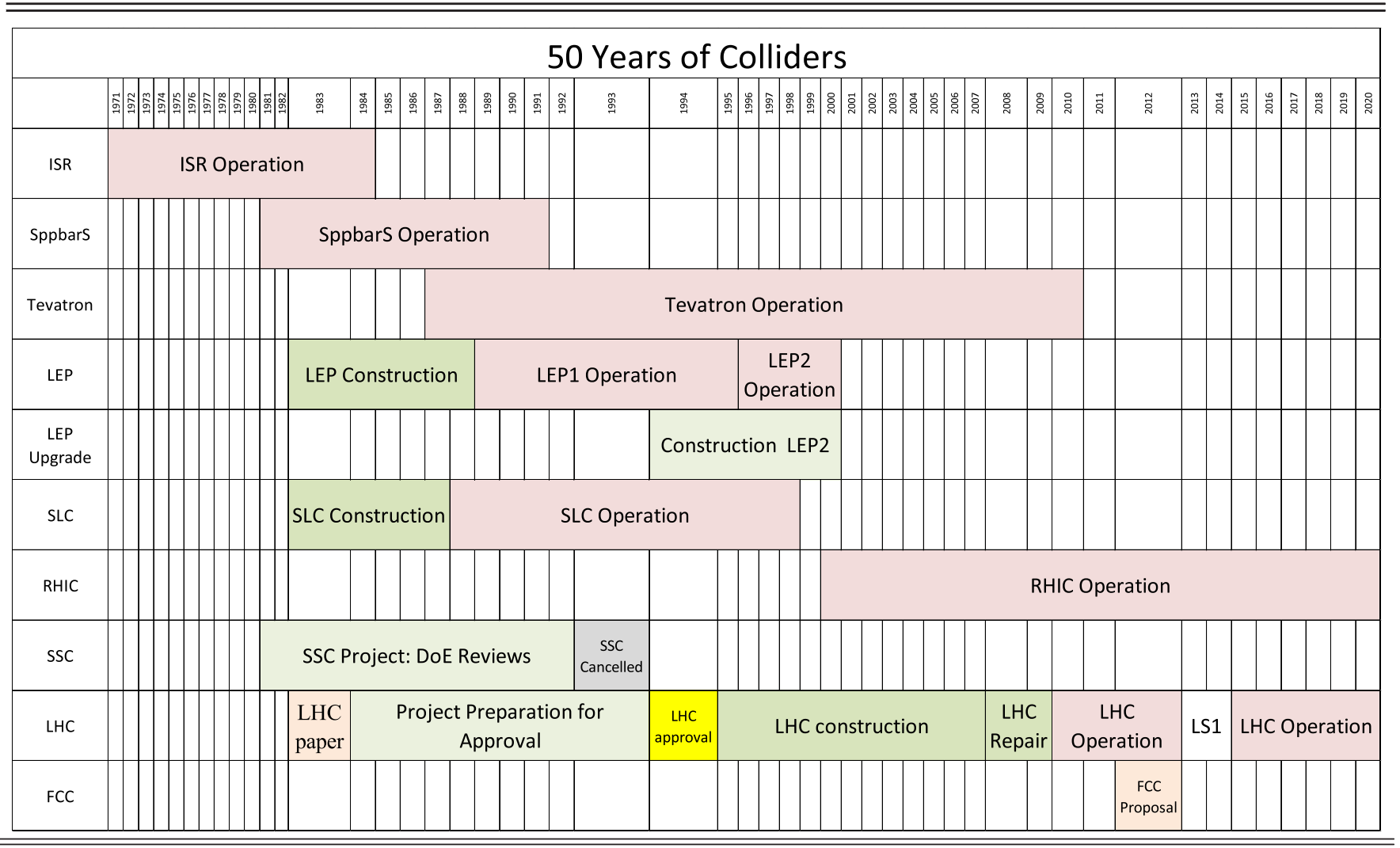


[1] E. Rutherford, Address of the president, Proc. R. Soc. A 117, 300 (1928).

[2] E. O. Lawrence, Method and apparatus for the acceleration of ions, U.S. Patent No. 1948384, granted for the cyclotron on 2 February 1934.

[3] M. K. Craddock, Cyclotrons and their applications, in Proceedings, 19th International Conference, Cyclotrons 2010, Lanzhou, China, September 6-10, 2010, edited by Y.-J. Yuan, L. Sheng, L.-J. Mao, and V. R. W. Schaa (Inst. Mod. Phys., Lanzhou, China, 2011), p. 452, CYCLOTRONS 10.

[4] Fifty Years of Synchrotrons-CERN, accelconf.web.cern.ch > PAPERS > ORAL.

[5] E. D. Courant, M. S. Livingston, and H. S. Snyder, The strong-focusing synchrotron-A new high energy accelerator, Phys. Rev. 88, 1190 (1952).

[6] N. C. Christofilos, Focusing system for ions and electrons, U.S. Patent No. 2, 736, 799, 1950.

[7] M. S. Livingston, High-Energy Accelerators (Interscience Publishers Inc., New York, 1954).

[8] C. Bernardini, AdA: The first electron-positron collider, Phys. Perspective 6, 156 (2004).

[9] P. J. Bryant, Dynamic compensation during stacking of the detuning caused by space charge effects, in Proceedings of the 1981 Particle Accelerator Conference: Accelerator Engineering and Technology, March 11-13, 1981, Washington, DC, Number 3 of IEEE transactions on nuclear science (1974), pp. 80-82, ISSN 0018-9499.

[10] J. Borer et al., Nondestructive diagnostics of coasting beams with Schottky noise, in Proceedings of the High-Energy Accelerator Conference, Stanford (1974), p. 53.

[11] K. Unser, A toroidal dc beam transformer with high resolution, in Proceedings of the 1981 Particle Accelerator Conference, Washington, DC (1981), pp. 2344-2346.

[12] P. G. Bramham, H. G. Carron, K. Hereward, W. S. Hübner, and L. Thorndahl, Stochastic cooling of a stored proton beam, Nucl. Instrum. Methods 125, 201 (1975).

[13] S. van der Meer, Stochastic damping of betatron oscillations in the ISR, Internal Report No. CERNISR-PO-7231, 1972.

[14] P. J. Amaldi, P. Bryant, and K. H. Darriulat, 40th anniversary of the first proton-proton collisions in the CERN intersecting storage rings (ISR), Report No. CERN-2012004, 2012.
[15] S. Myers; Simulation of the beam-beam effect for electronpositron storage rings, Nucl. Instrum. Methods Phys. Res. 211, 263 (1983); also Report No. CERN ISR/RF/82-06, 1982.

[16] S. Myers, A. Degiovanni, and J. B. Farr, Future prospects for particle therapy accelerators, Review of Accelerator Science and Technology, edited by A. W. Chao and W. Chou (World Scientific, Singapore, 2014), Vol. 7.

[17] Report of the Department of Energy Review Committee on the conceptual design of the superconducting super collider, Report No. DOE/ER-0267, 1986.

[18] Report of the Department of Energy Office of Energy Research Review Committee on the site-specific conceptual design of the superconducting super collider, Report No. DOE/ER-0463P, 1990.

[19] S. Myers and E. Picasso, The CERN LEP collider, Sci. Am. 263, 54 (1990).

[20] S. Myers and E. Picasso, The design, construction and commissioning of the CERN large-electron-positron collider, Contemp. Phys. 31, 387 (1990).

[21] S. Myers, The LEP collider, from design to approval and commissioning, The sixth John Adams memorial lecture delivered at CERN on 26 November 1990, Report No. CERN 91-08.

[22] S. Myers, The LEP machine-Present and future, Phil. Trans. R. Soc. A 336, 191 (1991).

[23] R. Assmann, M. Lamont, and S. Myers A brief history of the LEP collider, Nucl. Phys. B, Proc. Suppl. 109B, 17 (2002); also Report No. CERN-SL-2002-009.

[24] Wolfgang Schnell (1929-2006), CERN Bulletin No. 43/ 2006, BUL-NA-2006-147; 43/2206; 44/2006.

[25] M. Bajko et al., Report of the task force on the incident of 19 September 2008 at the LHC, LHC Project Report No. 1168, 2009.

[26] S. Myers, Large hadron collider commissioning and first operation, Royal Society discussion meeting, Phil. Trans. R. Soc A 370, 859 (2012).

[27] S. Myers and C. Wyss, Prospects for energy and luminosity at LEP2, Report No. CERN 96-01, Vol. 1, p. 26.

[28] O. Brüning, H. Burkhardt, and S. Myers, The large hadron collider, Prog. Part. Nucl. Phys. 67, 705 (2012).

[29] O. Brüning, B. Goddard, M. Mangano, S. Myers, L. Rossi, E. Todesco, and F. Zimmerman, High energy LHC document prepared for the European HEP strategy update, Report No. CERN-ATS-2012-237, 2012. 